\title{
Ten-m2 Is Required for the Generation of Binocular Visual Circuits
}

\author{
Timothy R. Young, ${ }^{1}$ Michael Bourke, ${ }^{1}$ Xiaohong Zhou, ${ }^{2}$ Toshitaka Oohashi, ${ }^{2}$ Atomu Sawatari, ${ }^{1 \star}$ Reinhard Fässler, ${ }^{2 \star}$ \\ and Catherine A. Leamey, ${ }^{1 *}$ \\ ${ }^{1}$ Discipline of Physiology, School of Medical Sciences and Bosch Institute, University of Sydney, New South Wales, 2006 Australia and ${ }^{2}$ Department of \\ Molecular Medicine, Max-Planck Institute for Biochemistry, D-82152 Martinsried, Germany
}

Functional binocular vision requires that inputs arising from the two retinae are integrated and precisely organized within central visual areas. Previous studies have demonstrated an important role for one member of the Ten-m/0dz/teneurin family, Ten-m3, in the mapping of ipsilateral retinal projections. Here, we have identified a distinct role for another closely related family member, Ten-m2, in the formation of the ipsilateral projection in the mouse visual system. Ten-m 2 expression was observed in the retina, dorsal lateral geniculate nucleus (dLGN), superior colliculus (SC), and primary visual cortex (V1) of the developing mouse. Anterograde and retrograde tracing experiments in Ten-m2 knock-out (KO) mice revealed a specific decrease in ipsilateral retinal ganglion cells projecting to dLGN and SC. This reduction was most prominent in regions corresponding to ventral retina. No change in the topography of ipsilateral or contralateral projections was observed. While expression of a critical ipsilateral fate determinant, Zic2, appeared unaltered, a notable reduction in one of its downstream targets, EphB1, was observed in ventral retina, suggesting that Ten-m2 may interact with this molecular pathway. Immunohistochemistry for c-fos, a neural activity marker, revealed that the area of V1 driven by ipsilateral inputs was reduced in K0s, while the ratio of ipsilateral-to-contralateral responses contributing to binocular activation during visually evoked potential recordings was also diminished. Finally, a novel two-alternative swim task revealed specific deficits associated with dorsal visual field. These data demonstrate a requirement for Ten-m 2 in the establishment of ipsilateral projections, and thus the generation of binocular circuits, critical for mammalian visual function.

\section{Introduction}

Functional binocular vision requires the precise integration and balance of inputs arising from the two eyes. This organization is critical for the appropriate processing of visual information, particularly within the primary visual cortex (V1). In mice, the majority of retinal ganglion cells (RGCs) project contralaterally, crossing the midline at the optic chiasm (OC). In contrast, the ipsilateral projection is much smaller $(\sim 3 \%)$ and is established almost exclusively from ventrotemporal (VT) retina (Dräger, 1985). Following partial decussation at the OC, axons project toward their major targets: the superior colliculus (SC) and the dorsal lateral geniculate nucleus (dLGN), which serves as a relay for inputs to V1.

Visuotopic alignment of inputs within these areas argues for the existence of eye-specific guidance mechanisms (Leamey et al.,

\footnotetext{
Received Oct. 5, 2012; revised May 3, 2013; accepted May 4, 2013.

Author contributions: T.R.Y., A.S., R.F., and C.A.L. designed research; T.R.Y., M.B., X.Z., T.O., A.S., R.F., and C.A.L. performed research; T.R.Y., A.S., and C.A.L. analyzed data; T.R.Y., A.S., and C.A.L. wrote the paper.

This work was supported by an National Health and Medical Research Council project grant and a University of Sydney research support grant to C.A.L. and in part by a Grant-in-Aid for Scientific Research on Innovative Areas (No. 24107516) to T.0. from the Ministry of Education, Culture, Sports, Science, and Technology (MEXT) of Japan. We acknowledge the use of the Bosch Institute Molecular Biology and Advanced Imaging facilities.

${ }^{*}$ A.S., R.F., and C.A.L. contributed equally to this work

Correspondence should be addressed to Catherine Leamey, Physiology, F13, University of Sydney, New South Wales 2006, Australia. E-mail: cathy@physiol.usyd.edu.au.

DOI:10.1523/JNEUROSCI.4708-12.2013

Copyright $\odot 2013$ the authors $\quad 0270-6474 / 13 / 3312490-20 \$ 15.00 / 0$
}

2009). Recent studies have demonstrated several molecular mechanisms linked to eye-specific pathways. Of note, Zic2 has been revealed to be a critical determinant of ipsilateral fate. Zic2 is expressed by uncrossed RGCs during their formation (Herrera et al., 2003), and regulates expression of molecules that mediate an ipsilateral trajectory, including the EphB1 receptor (GarcíaFrigola et al., 2008; Lee et al., 2008). This facilitates the repulsion of ipsilateral RGC axons away from the EphrinB2-rich midline (Williams et al., 2003). Other factors are thought to control contralateral fate, and may interact with and/or complement ipsilateral determinants (Pak et al., 2004; Williams et al., 2006; Tian et al., 2008).

The Ten-m/Odz/teneurin genes encode a highly conserved family of four transmembrane glycoproteins (for review, see Young and Leamey, 2009). We previously identified a role for one family member, Ten-m3, in the targeting of ipsilateral retinal projections in mice (Leamey et al., 2007; Merlin et al., 2013). Deletion of Ten-m3 leads to profound visual deficits, resulting from mismatched input from the two eyes (Leamey et al., 2007; Merlin et al., 2013). While the Ten-m family displays a high degree of homology between its members (Oohashi et al., 1999), no in vivo function has been described for any other mouse Ten-m to date. As this family has roles in regulating neural connectivity in vertebrate (Zhou et al., 2003; Li et al., 2006; Kenzelmann et al., 2008; Leamey et al., 2008) and invertebrate (Hong et al., 2012; Mosca et al., 2012) species, and multiple members are expressed highly in the devel- 
oping visual pathway (Leamey et al., 2008), we hypothesized that other Ten-ms may have specific, complementary roles in visual development.

Here, we investigated the role of Ten-m2, the family member with the highest homology to Ten-m3, in the mouse visual pathway. We found that Ten-m2 is expressed in key visual structures early in development. In its absence, ipsilateral projections were reduced, most notably from ventral retina, associated with diminished EphB1 expression. Contralateral projections from this region were expanded but displayed normal topography and segregation. Functional studies revealed a reduction in ipsilaterally driven activity within $\mathrm{V} 1$ as well as an inability to discriminate between dorsally located visual stimuli. These demonstrate a key functional role for Ten- $\mathrm{m} 2$ in the formation of binocular circuits which complements that of Ten-m3.

\section{Materials and Methods}

\section{Animals}

All experiments were performed on mice of both genders. For expression studies, C57BL/6 mice were obtained from timed-pregnant animals sourced from Animal Resources Centre (ARC; Perth, Australia). To generate a Ten-m2 knock-out (KO) mouse strain, genomic 129sv DNA of 11 $\mathrm{kb}$ length was subcloned into a pBluescript plasmid and a neomycinresistance cassette was inserted into exon 5 of the Ten-m2 gene, which encodes for the transmembrane region of the protein (Oohashi et al., 1999). This targeting vector was electroporated into R1 embryonic stem (ES) cells to generate 25 clones with a targeted disruption of the Ten-m2 gene. Two targeted ES clones were injected into host blastocyst to generate germ-line chimeras. Subsequent founder chimeras were backcrossed onto a C57BL/6 background and heterozygotes were obtained for further breeding. Genotyping was performed from DNA obtained by tail biopsy, using Southern blot with an external probe and standard PCR protocols using two genomic primers (sense: $5^{\prime}$-CTGTTCAGCAGCTCTTCTCC3'; antisense: $5^{\prime}$-AGCCTCTGGCCTCTTAATTC-3') and one primer from the neomycin-resistance cassette ( $5^{\prime}$-CGACATTCAACAGACCTT GC- $\left.3^{\prime}\right)$. Successful knockdown of the protein was confirmed by Western blotting and immunohistochemistry on KO brain tissue using antibodies generated against the extracellular domain of Ten-m2 (Zhou et al., 2003). For the visual discrimination task, several age-matched C57BL/6 mice (ARC) were used along with wild-type (WT) and KO littermates obtained from Ten- $\mathrm{m} 2$ breeding. All experiments were performed on adult $(>12$ weeks) mice, unless otherwise stated. KOs were generated and housed under the guidelines provided by the Bavarian government. All other procedures were approved by Animal Ethics Committee of the University of Sydney and conformed to National Health and Medical Research Council guidelines.

\section{In situ hybridization and immunohistochemistry}

The mice were killed via an intraperitoneal injection of sodium pentobarbital $(>100 \mathrm{mg} / \mathrm{kg})$. In situ hybridization was performed on $15-\mu \mathrm{m}$-thick freshfrozen cryostat sections using digoxigenin-labeled riboprobes, as described previously (Leamey et al., 2007). The following primers were used to PCRamplify cDNA fragments ( $\sim 200 \mathrm{bp}$ ), which were then each subcloned into a pGEM-T expression plasmid (Promega): Ten-m2 forward (fwd), $5^{\prime}$-CTCT ATGACCCCCTCACCAA-3'; Ten-m2 reverse (rev), 5' -CCAGCTCTTCA CGTCTGTCA-3'; EphB1 fwd, 5'-GATTTTGGGGGAAGGTTGAT-3'; EphB1 rev, 5'-ATGGTTTCTGGGAACACTGC-3'. Sense and antisense riboprobes were synthesized using in vitro transcription (Promega). Fluorescein-conjugated tyramide signal amplification (TSA; PerkinElmer) was used to develop the reaction. Sections were counterstained with DAPI and coverslipped with anti-fade reagent (Invitrogen).

Immunohistochemistry for Ten-m expression was performed using $15-\mu \mathrm{m}$-thick fresh-frozen cryostat sections, as described previously (Leamey et al., 2007). Sections were fixed with $4 \%$ (w/v) paraformaldehyde (PFA) and blocked with 5\% (v/v) normal goat serum (NGS; Vector Laboratories) and $2.5 \%(\mathrm{w} / \mathrm{v})$ bovine serum albumin (Roche) plus $0.1 \%$ (v/v) Triton X-100 (Sigma), before incubation with primary antibodies, overnight at $4^{\circ} \mathrm{C}$. Zic2 immunohistochemistry was performed on freshly dissected retinae (E16.5), blocked with $10 \%$ NGS plus $0.1 \%$ Triton $\mathrm{X}-100$, and reacted as whole mounts. Primary antibodies were used as follows: rabbit polyclonal anti-mouse Ten-m2, 1 in 200 dilution (Zhou et al., 2003) and rabbit anti-Zic2, 1 in 10,000 dilution (Brown et al., 2003). For Ten-m2 immunostaining, primary antibody incubation was followed by a biotinylated goat anti-rabbit IgG secondary antibody ( 1 in 200 dilution; Vector Laboratories) and then developed using ABC (Vector Laboratories) and a fluorescein-TSA reaction (PerkinElmer). Control experiments were either performed using Ten-m2 KO sections or by incubating with secondary antibody alone. Zic2 primary antibody incubation was followed by an Alexa Fluor 594-conjugated goat anti-rabbit IgG secondary antibody (1 in 200 dilution; Invitrogen). Retinae were then flat-mounted and visualized on glass microscope slides. All antibodies were diluted in $2 \%$ NGS with $0.1 \%$ Triton X-100.

\section{Tracer injections}

All surgical procedures were performed under anesthesia induced and maintained by inhalation of $2-4 \%(\mathrm{v} / \mathrm{v})$ isoflurane in oxygen. For anterograde labeling from the retina, intraocular injections were made into each eye of adult WT and KO littermates ( postnatal day 28-30; P28-30) using $1 \mu \mathrm{l}$ of $1 \%(\mathrm{w} / \mathrm{v}$; in $0.1 \mathrm{M}$ phosphate buffer; $\mathrm{PB})$ cholera toxin subunit B (CTB; Invitrogen) conjugated to either Alexa Fluor 488 (green) or Alexa Fluor 594 (red). After $24-48 \mathrm{~h}$, animals were killed with a lethal dose of sodium pentobarbital $(>100 \mathrm{mg} / \mathrm{kg}$ ) and perfused transcardially with $0.9 \%(\mathrm{w} / \mathrm{v})$ saline containing $10 \mathrm{U} / \mathrm{ml}$ heparin sodium (SigmaAldrich), followed by $4 \%$ PFA in $0.1 \mathrm{M} \mathrm{PB}$. Brains were dissected and postfixed overnight at $4^{\circ} \mathrm{C}$. Coronal sections (60 $\mu \mathrm{m}$ thick) were made through the dLGN and SC with a vibrating microtome (1000 Plus system; Vibratome). To study retinocollicular projections in more detail, some animals were killed as above and were lightly fixed by perfusion with $2 \%$ PFA. SCs were dissected and postfixed overnight at $4^{\circ} \mathrm{C}$ while being flattened between two glass microscope slides separated by a height equivalent to five coverslips $(\sim 1 \mathrm{~mm})$. After cryoprotection in $30 \%$ $(\mathrm{w} / \mathrm{v})$ sucrose, flattened SCs were sectioned tangentially $(150 \mu \mathrm{m})$ to the pial surface using a freezing microtome (Leica).

For retrograde labeling from the thalamus, small volumes of 5\% (w/v) wheat germ agglutinin conjugated to horseradish peroxidase (WGAHRP; Sigma-Aldrich) in $0.9 \%$ saline were pressure injected unilaterally into dLGN using a pneumatic PicoPump machine (World Precision Instruments). Using stereotaxic coordinates, multiple injections (4-5 injections, spaced $100 \mu \mathrm{m}$ apart, and centered around $2.2 \mathrm{~mm}$ lateral, 1.5 $\mathrm{mm}$ rostral to lambda; depth $2.5 \mathrm{~mm}$ ) were performed to ensure a complete fill of the nucleus. After $48 \mathrm{~h}$, animals were killed as above and lightly fixed by perfusion with $2 \%$ PFA. Retinae were dissected and reacted as whole mounts, while coronal sections $(100 \mu \mathrm{m})$ were cut through the dLGN using a vibrating microtome (1000 Plus system; Vibratome). Reaction product was developed with a tetramethyl benzidine reaction using standard techniques (Mesulam, 1978).

To investigate potential changes in topography, focal injections of the lipophilic carbocyanine dye, dioctadecyl-3-3-3'-3' -tetramethyl-indocarbocyanine perchlorate (DiI; Invitrogen) were made into ventral retina of anesthetized mice at P13. A working DiI preparation was made by diluting a $10 \%$ (w/v) DiI solution (in dimethylformamide) to a final concentration of $4 \%$ with $10 \%(\mathrm{w} / \mathrm{v})$ sucrose. Following opening of the palpebral fissure, a small incision in the sclera was made to expose the retina. Using a fine glass micropipette, a small volume of DiI was pressure injected into ventral retina, and the animal allowed to recover. After 1-2 d transport, animals were killed and perfused as above. Retinae were dissected and flat mounted onto glass microscope slides, while brains were embedded in 3\% (w/v) agarose, and coronal sections $(100 \mu \mathrm{m})$ were cut through the dLGN using a vibrating microtome.

Image analysis. All images were obtained using either an Axioplan 2 fluorescence microscope attached to an AxioCam HR digital monochrome camera, and acquired with AxioVision software (Zeiss), or bright-field illumination using a Nikon E800 microscope and digital camera (Nikon). Analyses were performed using ImageJ software (National Institutes of Health (NIH, Bethesda, MD).

For anterograde labeling of retinogeniculate projections, the perimeter of each dLGN was outlined using the freehand tool, taking care to 
exclude the neighboring optic tract, intergeniculate leaflet, and ventral LGN. For each section, background was subtracted from the image channel containing ipsilateral label (rolling ball function, radius 250), which was then thresholded at a constant arbitrary value (grayscale value $30-$ 255). The proportion of the area taken up by ipsilateral axons within the dLGN was calculated as a percentage of total dLGN area. Comparisons between animals were performed by alignment, according to both dLGN shape and size, of 11 consecutive sections along the rostrocaudal extent of the nucleus for each animal. Rostral and caudal extremes of the dLGN were excluded due to higher variability in label signal and background. Animals in which the contralateral label did not completely fill the dLGN were excluded from analyses. To determine the degree of segregation of retinal inputs within the dLGN, a threshold analysis was performed in a manner similar to that described previously (Muir-Robinson et al., 2002; Rebsam et al., 2009).

Horizontal SC sections were analyzed by calculating the ipsilateral area percentage in each SC hemisphere. For each flattened SC, ipsilateral label was seen at a number of depths (usually $0-450 \mu \mathrm{m}$ ). Background was subtracted from grayscale images (rolling ball function, radius 300) and thresholded images were aligned for depth using the caudal end of the pretectum as a fiducial. In images that contained signal, ipsilateral area was determined in each section and percentage of total SC area was calculated. Composite heatmaps were generated with pixels from each ipsilateral channel summed into 100 bins across both the mediolateral and rostrocaudal axes of each SC. Signal plots for each SC were averaged across genotypes and a heatmap was generated using the statistical program, R (R Foundation for Statistical Computing).

For WGA-HRP injections, only those animals in which label filled the entire contralateral retina and dLGN were used in analyses. Labeled cells in the ipsilateral retina (VT retina) were counted using Neurolucida software (MBF Bioscience). Total retinal area, and that occupied by retrogradely labeled cells, were also measured and used to calculate percentage area and cell density, respectively. The arc between the nasal fiducial mark and the temporal and ventral extremes of the ipsilaterally labeled ventrotemporal crescent (VTC) were measured around the perimeter of the retina and divided by the total retinal circumference to give the temporal and ventral arc ratios, respectively using Image (NIH, Bethesda, $\mathrm{MD})$. The relative length of the arc associated with the ipsilateral label was similarly determined to give the ipsilateral arc ratio. The width of the ipsilaterally labeled VTC was measured across its radial axis at the region of its maximum extent and divided by the radius of the retina in that region to give the ipsilateral radial ratio.

A semiquantitative analysis of EphB1 expression in E16.5 retina was performed using grayscale fluorescence images obtained from in situ hybridization experiments. After subtracting background and thresholding images as described above, pixel area and length of the retina occupied by label was measured and further used to calculate density of EphB1 signal.

$c$-fos immunohistochemistry. An assessment of neuronal activity in V1 was performed using c-fos immunohistochemistry, as described previously (Merlin et al., 2013). Mice were anesthetized as above and an intraocular injection $(1 \mu \mathrm{l})$ of a $3 \mathrm{~mm}$ solution of tetrodotoxin (SigmaAldrich) was made into the posterior chamber of one eye, which was immediately rinsed with $0.9 \%$ saline; the animals were then allowed to recover. After checking for absence of pupillary reflexes, mice were placed in a darkened room for $16 \mathrm{~h}$. Animals were exposed to light for $2 \mathrm{~h}$, killed as above, followed by transcardial perfusion with $0.9 \%$ saline and $4 \%$ PFA. Cortices were dissected and postfixed while being flattened between two microscope slides, as described above. Following cryoprotection, flattened cortices were sectioned $(40 \mu \mathrm{m})$ tangential to the pial surface using a freezing microtome. Sections were wet mounted onto glass slides using $0.1 \mathrm{MPB}$ and were transilluminated to identify V1 borders (Trevelyan et al., 2007), and imaged under a Leica M420 macroscope (Leica Microsystems) using an Olympus DP70 digital camera. Sections were then demounted and processed for c-fos immunostaining. Endogenous peroxidase activity was quenched with $0.3 \%(\mathrm{v} / \mathrm{v})$ hydrogen peroxide and sections were blocked with $2 \%$ NGS plus $0.3 \%$ Triton X-100, before incubation with rabbit anti-c-fos antibody ( 1 in 5000 dilution; Santa Cruz Biotechnology). Primary incubation was followed by a biotinylated goat anti-rabbit IgG secondary antibody ( 1 in 200 dilution;
Vector Laboratories). Reaction product was developed using ABC (Vector Laboratories) and a cobalt chloride-enhanced DAB reaction. Sections were imaged under bright-field illumination using a Nikon E800 microscope and digital camera. Sections containing layer IV of V1 were analyzed, with V1 boundaries being identified by overlaying with images obtained by transillumination. The level of c-fos expression within V1 was then determined by first removing background and thresholding images using Image (NIH, Bethesda, MD). To generate composite heatmaps, thresholded pixels were summed into 100 bins across both the mediolateral and rostrocaudal axes of ipsilateral V1. Normalized signal plots for each animal were averaged across genotypes and a heatmap was generated using the statistical program, R (R Foundation for Statistical Computing).

Visually evoked potential recordings. All experiments were performed in the dark on adult WT and KO mice, in a similar manner to that described by Porciatti et al. (1999). Animals were anesthetized with an intraperitoneal injection $(\sim 1.5 \mathrm{~g} / \mathrm{kg})$ of urethane (Sigma-Aldrich) in $0.9 \%$ saline, followed by maintenance doses $(0.1 \mathrm{~g} / \mathrm{kg})$ administered bihourly. Once adequate anesthesia was achieved, mice were positioned 75 $\mathrm{cm}$ from a matte white screen $(170 \mathrm{~cm}$ wide $\times 127 \mathrm{~cm}$ high $)$ in a modified rodent stereotax (Kopf). A craniectomy (area $\sim 1 \mathrm{~mm}^{2}$; centered at 3.0 $\mathrm{mm}$ lateral and $0.5 \mathrm{~mm}$ posterior to lambda) was performed to expose the binocular region of V1. Recordings were made using resin-coated tungsten electrodes ( $\sim \mathrm{M} \Omega$ at $1 \mathrm{kHz}, 10 \mathrm{nA}, 1 \mathrm{~mm}$; FHC), mounted in a headstage (Digitimer) attached to a micromanipulator (World Precision Instruments), advanced to a depth of $500 \mu \mathrm{m}$ to target layer IV. Signals were amplified $(10,000 \times)$, bandpass filtered $(0.5-120 \mathrm{~Hz})$ using NeuroLog hardware (Digitimer, UK), and waveforms recorded using EXPO (software written by Peter Lennie, New York University, New York, NY).

Visual stimuli generated in EXPO were displayed using an LCD projector (EMP-TW700; Epson). Stimuli consisted of drifting horizontal sine-wave gratings (drift rate: 2 cycles/s, spatial frequency: 0.1 cycles/ degree), presented across the azimuth of the binocular and unilateral monocular visual fields $\left(-7.5-82.5^{\circ}\right)$ as six nonoverlapping vertical columns $\left(15 \times 70^{\circ}\right)$, contralateral to the recorded cortical hemisphere. This arrangement meant that the central-most column was centered directly in front of the animal. Each vertical column, plus a blank control (500 ms duration with $500 \mathrm{~ms}$ interstimulus period) was presented in a pseudorandom seven-trial sequence. A total of 60 trials worth of responses were recorded for each of the six visual locations in this manner.

Recordings were made following a sequence of (1) binocular, (2) contralateral, (3) ipsilateral, and (4) binocular viewings, in which the appropriate eyes were covered/uncovered with adjustable blinders. Following each cortical penetration, the electrode tip was removed, dipped in either green or red fluorescent microbeads (Lumafluor), and reinserted into the same site to mark its position.

Upon the completion of recordings, animals were killed, perfused, and the recorded cortex dissected and flattened, as described above. Tangential sections $(40 \mu \mathrm{m})$ were cut using a freezing microtome and transilluminated to identify V1 borders (see above). The location of electrode recording sites were then imaged using an Axioplan 2 fluorescence microscope attached to an AxioCam HR digital monochrome camera, and acquired using AxioVision software (Zeiss).

Analysis of visually evoked potential recordings. All recordings confirmed to be from the binocular region of V1 (as determined by transillumination/fluorescent images) were included in the analysis. Coronal sections in sample experiments ( $n=4$ for WT; $n=2$ for KO) indicated that the electrode tip was consistently targeted to layer IV of the cortex for both genotypes. Further, no notable difference in cortical thickness between genotypes was found. For each recording site, the visual location that elicited maximal binocular visually evoked potential (VEP) responses (measured peak to trough; see below) were identified and further assessed across contralateral and ipsilateral viewings. Sets of recordings (i.e., corresponding binocular, contralateral, ipsilateral traces) were considered reliable, and thus included in the analysis, only if the amplitude of the second binocular responses were within 1 SD of the first binocular response. This was done to select against gain/loss of responsiveness and/or shifts in electrode position during recordings. 
The following VEP trace parameters were included in the analysis: amplitude (for peak, trough, and peak to trough), latency (to peak and to trough), slope (to peak), slope (peak to trough), and total area under the curve (AUC). Amplitude: For each response, a baseline value was calculated based on the first $10 \mathrm{~ms}$ of each trace and was subtracted to determine values for the peak (maximum positive deflection) and trough (maximum negative deflection). Latency: For consistency, latency was calculated as the time to peak or time to trough. Slope (to peak): The slope of the positive component of the response was determined from values taken from the 20 to $80 \%$ range of the peak response, to minimize the effect of noise in each trace. Slope (peak to trough): The slope of the negative component was calculated from values taken from the 20 to $80 \%$ range of the peak-to-trough response. Total AUC: The absolute sum of all sampled points that fell either above or below baseline (see above) was calculated and the absolute sum of the blank control subtracted to determine an effective AUC.

\section{Visual discrimination task}

Adult mice were subjected to a forced-choice visual discrimination task that tested their ability to discriminate dorsally located visual stimuli, adapted from the behavioral paradigm developed by Prusky et al. (2000).

Apparatus. A glass aquarium $(140 \mathrm{~cm}$ long $\times 80 \mathrm{~cm}$ wide $\times 52 \mathrm{~cm}$ high) was filled to a depth of $15 \mathrm{~cm}(\sim 160 \mathrm{~L})$ with water $\left(24-25^{\circ} \mathrm{C}\right)$ rendered opaque by the addition of a mixture of white, nontoxic, watersoluble paint $(50 \mathrm{ml})$ and black food dye $(3 \mathrm{ml})$. The front wall of the aquarium was extended to a height of $110 \mathrm{~cm}$ using white/translucent Perspex, onto which visual stimuli were displayed using an LCD projector (EMP-TW700; Epson). Visual stimuli $(30 \times 30 \mathrm{~cm}$, corresponding to $22 \times 22^{\circ}$ at $75 \mathrm{~cm}$ from the front end of the pool; EXPO written by Peter Lennie, New York University), consisting of either vertical (positive stimulus) or horizontal (negative stimulus) static sine-wave gratings (spatial frequency: 0.123 cycles/degree), were projected from a distance of 3 and $1 \mathrm{~m}$ above the height of the water. A matte black Perspex middle divider ( $110 \mathrm{~cm}$ high $\times 45 \mathrm{~cm}$ long) extended out from the front wall and partitioned the front end of the aquarium into two end zones. A submerged escape platform $(37 \mathrm{~cm}$ long $\times 13 \mathrm{~cm}$ wide $\times 14 \mathrm{~cm}$ high, $1 \mathrm{~cm}$ below water surface) was hidden in one of the end zones, always being located on the side containing the positive stimulus. With the exception of the front end, the walls of the aquarium were covered with matte black Perspex to minimize reflections. Furthermore, compressed air was bubbled steadily underneath the water surface to provide sufficient agitation to reduce potential cues arising from surface reflections, without impeding swimming ability. The back walls of the aquarium were angled inward to form a trapezoidal shape and were continuous with a central release chute $(20 \mathrm{~cm}$ long $\times 10 \mathrm{~cm}$ wide $)$ that directed an animal toward the front end upon release. The entire experiment was performed in the dark with the only light originating from the projected stimuli. Animals' performances were monitored and digitally captured via an infrared camera mounted directly above the apparatus.

Procedure. The task was run over consecutive days (usually $\sim 3$ weeks in total) and involved three phases: pretraining, training, and dorsal discrimination testing. On each day of testing, animals were involved in two sessions of 10 trials each, separated by a 60 min rest period. Briefly, for each trial, the location of the hidden escape platform and positive stimulus was alternated between each end zone using a pseudorandom sequence based on previously described rules (Prusky et al., 2000). For each cohort, mice were tested with interleaved trials, being placed within the release chute and given $90 \mathrm{~s}$ to locate the platform, on which they were allowed to remain for $10 \mathrm{~s}$ before being removed and returned to their housing cage. The plane perpendicular to and situated at the end of the middle divider acted to set the "decision " line across which an animal was deemed to have made a "choice " based on the presented stimuli. Incorrect decisions were recorded if the animal crossed the decision line into the side containing the negative stimulus (nonplatform side) during any part of the trial. An incorrect choice was also recorded if an animal was not able to locate the platform in the allocated $90 \mathrm{~s}$, after which it was guided to swim onto the platform and allowed to stay for $10 \mathrm{~s}$. Incorrect choices were followed immediately by a single repeat trial to act as a "penalty" and reinforce the task. Correct decisions were recorded only if the animal was able to locate and remain on the platform for $10 \mathrm{~s}$, without entering the negative stimulus side at any point during the trial.

During pretraining, a single vertical grating stimulus was presented on either side of the front wall, located $5 \mathrm{~cm}$ above the surface of the water. Mice were gradually trained to swim toward and associate the visual stimulus with the escape platform. Initially, animals started on the same side as the platform/stimulus, being released directly in front from a distance of $10 \mathrm{~cm}$. After repeating on the opposite side, the release distance from the platform was systematically increased by increments of $\sim 20 \mathrm{~cm}$ until mice started from the central release chute and reliably swam toward the end zone (typically achieved by the end of the first session). From this stage, performance was measured as the percentage of correct choices during a 10 trial session. Animals that performed at $\geq 80 \%$ for two consecutive sessions (with the last above-threshold session required to occur after an overnight consolidation period) were moved on to the training phase of the task (typically achieved by the end of the third session).

In the training phase, mice learned to discriminate between two different stimuli (vertical vs horizontal gratings), with vertical gratings (positive) being associated with the escape platform. The same $\geq 80 \%$ correct performance threshold was used as criteria to select animals that proceeded on to the next phase of the task.

For dorsal discrimination, the ability of the mice to perform the previously trained discrimination task but with stimuli in more dorsally located positions was tested. The locations of both visual stimuli were sequentially raised by increments of $4^{\circ}$ whenever an animal performed at or above a $70 \%$ correct threshold (assessed over one session) for a tested stimulus height. If an animal performed below this threshold, stimuli were returned to their original level used for the training phase, and dorsal discrimination was systematically retested. If an animal consistently fell below performance threshold at a previously tested height, the animal was removed from testing and the highest location of the stimuli before which performance fell below $70 \%$ was regarded as its limit for dorsal discrimination in this task.

For some animal cohorts, mice were retrained to the original training stimuli and visual acuity was assessed using a similar protocol as above to obtain a spatial frequency threshold (Prusky et al., 2000). During the initial stages of testing ( $0.123-0.251$ cycles/degree), spatial frequency of both stimuli was increased for each trial where a correct decision was made. Subsequently, the task was made more stringent with animals being required to make multiple consecutive correct decisions before increasing the spatial frequency (two correct for $0.283-0.318$ cycles/degree; three correct for $0.352-0.385$ cycles/degree; four correct for $\geq 0.419$ cycles/degree). Upon determining an initial acuity threshold using this method, the animal's performance at the same spatial frequency level was assessed over 10 trials. If performance fell below 70\%, the spatial frequency was lowered to the previous frequency and performance was subsequently assessed over 10 further trials. With this procedure, the time an animal spent testing at easier spatial frequencies was minimized, while testing near the animal's acuity threshold could be commenced with less chance of a bias developing over this period. If an animal consistently fell below performance threshold at a previously tested spatial frequency, the animal was removed from testing and the highest frequency of the stimuli before which performance fell below $70 \%$ was regarded as its effective visual acuity in this task.

\section{Statistical analysis}

Graphs were constructed and data analyzed using either Microsoft Excel, SPSS software (IBM), or the statistical program, R. Error bars indicate SEM. Statistical analyses were performed using a two-tailed Student's unpaired $t$ test, multivariate ANOVA, Mann-Whitney $U$ test, or Fisher's exact test, where appropriate.

\section{Results}

\section{Ten-m2 is expressed during development in the mouse visual system}

To determine whether Ten-m2, the member of the teneurin family most closely related to Ten-m3 (Tucker et al., 2012), plays a role in the development of the mouse visual system, we first ex- 
amined its expression within key components of the early visual pathway (retina, SC, dLGN, and V1). Ten-m2 was found to be present in all these areas during the period in which axons project from the retina and innervate their targets (E14-P7). By combining our in situ hybridization and immunohistochemical data, we were able to confirm specific, corresponding patterns of expression of both mRNA and protein in the visual areas that we studied.

Using an antibody directed against the extracellular region of Ten-m2 (Zhou et al., 2003), cellular expression was observed primarily around somata and axonal tracts (Fig. 1). In the retina, immunostaining revealed that Ten-m2 was expressed prominently within the RGC layer. At E14, expression appeared highest within central retina (data not shown). By E16, however, immunostaining had expanded throughout the dorsoventral axis to become uniformly distributed across the retina (Fig. $1 A$ ). This pattern appears to match the central-to-peripheral time course for the generation of RGCs (Dräger, 1985). Real-time reverse transcription (RT)-PCR analysis confirmed the absence of graded expression for Ten- $\mathrm{m} 2$ across the dorsoventral and nasotemporal axes of the retina at P0 (data not shown). We also observed that by $\mathrm{P} 0$, the junction between the RGC layer and underlying inner plexiform layer (IPL) developed a prominent level of immunostaining (Fig. $1 A^{\prime}$, arrow). No staining was observed in the retina of Ten-m2 KO mice at E16 (Fig. $1 B$ ), confirming the specificity of the antibody.

Expression was maintained along the retinofugal pathway, with Ten-m 2 immunostaining observed on axons (as seen at E14 and E16) in the optic nerve, optic tract, and at the OC. When viewed in coronal sections around the OC, a higher level of expression was apparent in the dorsal/lateral regions of these axonal tracts (E16; Fig. 1C, arrowheads), potentially reflecting the temporal order observed for the location/laterality in which RGCs are generated in the retina (Bovolenta and Mason, 1987; Reese, 1987; Reese et al., 1992; Chan and Guillery, 1994). At the OC, Ten-m2 was notably absent in the region of radial glial cells, adjacent to the crossing RGC axons, suggesting that axonal repulsion/crossing in this area is unlikely to involve Ten- $\mathrm{m} 2$ interactions. Several areas proximal to the chiasm were also Ten-m2-positive: these regions, however, were generally dorsal and anterior to the optic tract and appeared to correlate with distinct areas such as the medial preoptic nucleus of the hypothalamus. Adjacent control sections showed no evidence of staining (Fig. 1D).

Ten-m2 expression was also observed in two major retinal targets, the dLGN and SC. Here, expression was uniform across the dorsomedial-ventrolateral axis of the dLGN, at all ages studied (E16-P7). This pattern appeared to be consistent in coronal sections along the rostrocaudal axis, further suggesting that expression was uniform throughout the nucleus (Fig. 1E). Corresponding patterns were observed using in situ hybridization (Fig. $1 E$ ) and immunohistochemistry (Fig. $1 G$ ). No staining was present in control sections (Fig. $1 F, H$ ). Ten-m2 mRNA (Fig. 1I) and immunoreactivity (Fig. $1 K$ ) were consistently expressed in the superficial, retinorecipient layers of the SC between P0 and P7, with no staining in corresponding control sections (Fig. $1 \mathrm{~J}, L$ ). No clear gradient of expression was found across either of the two major axes of the SC (E16-P7) in any of our experiments, including real-time RT-PCR analysis (data not shown). Expression of Ten-m 2 was further evident throughout the retinogeniculocortical pathway, being observed in areas of the neocortex that correspond to visual cortex (E16-P0). At P0, expression of Ten-m2 mRNA was seen in the deep part of the cortex using in situ hybridization (Fig. 1M) and by P7, could be identified in layers IV and $\mathrm{V}$ of primary visual cortex (V1) using immunohistochemistry (Fig. 1O). Staining was absent from control sections (Fig. $1 N, P)$.

\section{Anterograde tracing from the retina revealed distinct changes to ipsilateral projections in Ten-m $2 \mathrm{KO}$ mice}

Expression of Ten-m 2 in interconnected regions of the visual pathway suggested a possible role for this glycoprotein in the development of neural connectivity between these areas. To investigate this, a $\mathrm{KO}$ mouse strain was generated by targeted disruption within exon 5 of the Ten-m 2 gene (Fig. $2 A$ ). Southern blot analysis confirmed the success of the targeting strategy, with successful knockdown of protein expression demonstrated by Western blot (Fig. 2B). KO animals were viable and healthy into adulthood, maintaining normal body weights (WT: $29.0 \pm 1.0 \mathrm{~g}$, $n=13$; KO: $27.6 \pm 1.7 \mathrm{~g}, n=15 ; p=0.493$, Student's unpaired $t$ test). Further, they showed no obvious morphological abnormalities. Most significantly here, head shape and eye position appeared normal (Fig. 2C; see also below) and KOs displayed no evidence of developmental delays. The overall cytoarchitectural layout and size (as determined by Nissl staining) of visual areas of KO mice appeared normal when compared with WT mice (data not shown). To further assess the overall organization of the visual pathways of these mice, retinal projections were anterogradely labeled using intraocular injections of CTB conjugated to either Alexa Fluor 488 (green) or Alexa Fluor 594 (red) to reveal ipsilateral and contralateral projections terminating within the dLGN and SC.

\section{Retinogeniculate projections}

The general appearance of the retinogeniculate projection was largely similar between WT and KO mice (Fig. 3). In the dLGN, we found ipsilateral terminals occupied a stereotypical dorsomedial position within rostral sections, shifting slightly ventrolaterally and taking up a larger area of the nucleus in more caudal sections for both WTs and KOs (Fig. $3 A-F$ ). As reported for adult mice, contralateral projections occupied the remainder of the dLGN, and did not overlap with their ipsilateral counterparts (Muir-Robinson et al., 2002).

Interestingly, however, quantitative analysis revealed that the volume of the dLGN occupied by ipsilateral projections was slightly $(\sim 13 \%)$, but significantly, smaller in Ten-m2 KO mice, compared with WTs (mean ipsilateral volume percentage \pm SEM; WT: $12.77 \pm 0.28 \%, n=18$ dLGNs; Ten-m2 KO: $11.12 \pm$ $0.28 \%, n=16$; $p<0.001$, Student's unpaired $t$ test). Upon closer visual inspection, the size of the ipsilateral projection was notably smaller in rostral sections, though appeared unchanged more caudally in Ten-m2 KO dLGN (Fig. 3D-F).

To investigate these differences more thoroughly, a comparison of the ipsilateral terminal size across the rostrocaudal axis of the dLGN was performed using coronal sections through the nucleus (Fig. 3G-I). We found that ipsilateral area in Ten-m2 KOs was significantly reduced, compared with WTs; this was, however, limited to the rostral half of the dLGN (Fig. $3 G$, sections 7-10; WT, $n=18$ dLGNs; Ten-m2 KO, $n=16$; multivariate ANOVA; section (s) 6: $F_{(1,32)}=4.04, p=0.053$; $s 7: F_{(1,32)}=22.80$, $p<0.001$; s8: $F_{(1,32)}=31.48, p<0.001 ; s 9: F_{(1,32)}=38.75, p<$ 0.001 ; s10: $F_{(1,32)}=18.90, p<0.001$; all other sections $\left.p>0.1\right)$. Notably, these differences were observed in the absence of any changes in total dLGN area at any point across its rostrocaudal axis (Fig. $3 H$ ) or overall dLGN size (mean dLGN volume \pm SEM; WT: $1.98 \times 10^{-1} \pm 2.86 \times 10^{-3} \mathrm{~mm}^{3}$; Ten-m2 KO: $2.07 \times$ $10^{-1} \pm 5.91 \times 10^{-3} \mathrm{~mm}^{3} ; p=0.174$, Student's unpaired $t$ test). 


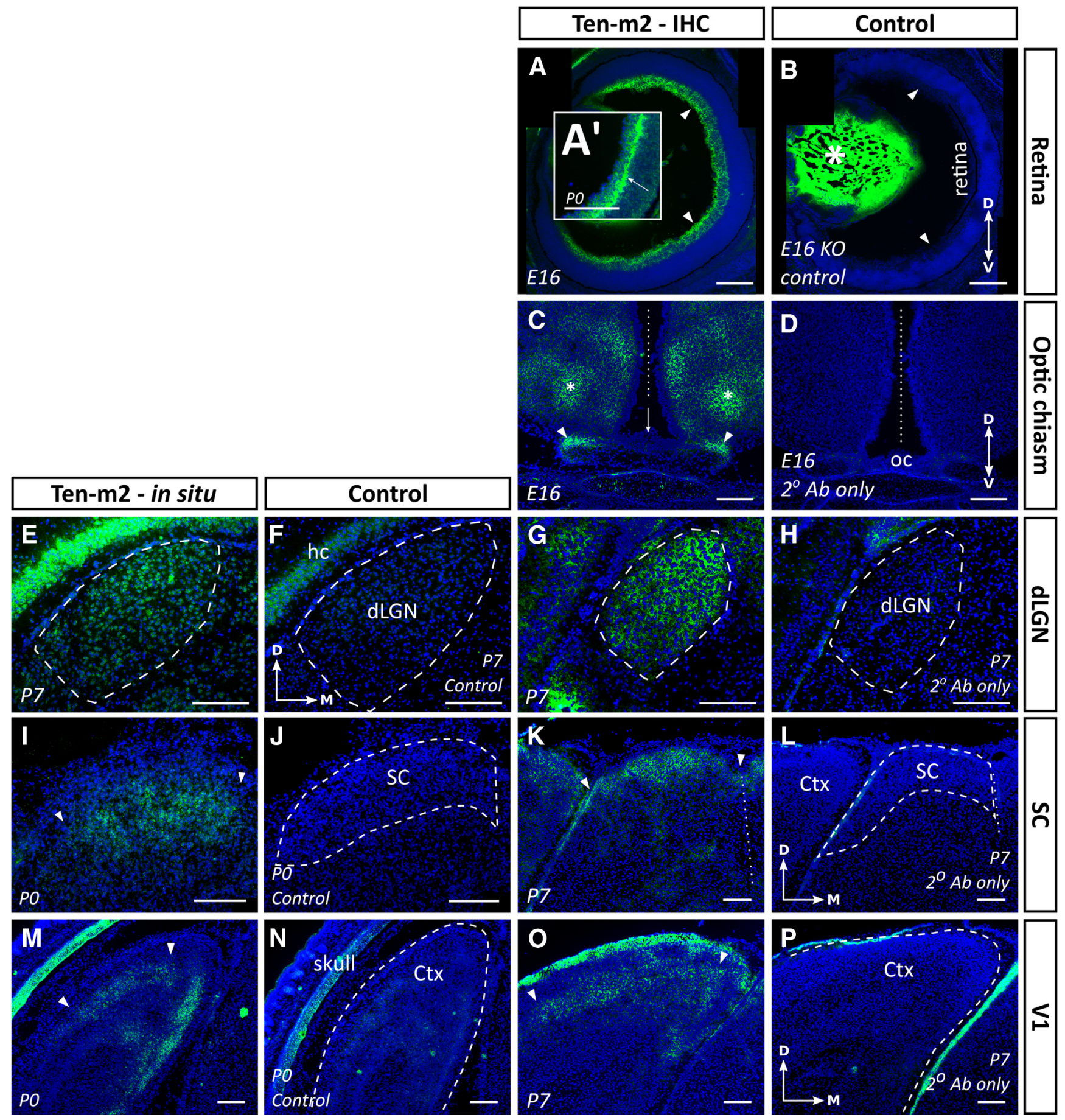

Figure 1. Ten-m2 is expressed in interconnected regions of the developing mouse visual system. Expression studies revealed the presence of Ten-m2 at multiple levels of the developing mouse visual system. Consistent expression patterns were seen using both in situ hybridization $(\boldsymbol{E}, \boldsymbol{F}, \boldsymbol{I}, \boldsymbol{J}, \boldsymbol{M}, \boldsymbol{N})$ and immunohistochemistry $(\boldsymbol{A}-\boldsymbol{D}, \boldsymbol{G}, \boldsymbol{H}, \boldsymbol{K}, \boldsymbol{L}, \mathbf{O}, \boldsymbol{P})$ in visual centers. $A, B$, Immunostaining for Ten-m2 in the retina was present in the RGC layer, including the region corresponding to the passage of fibers (arrowheads). By E16 expression was uniform across the dorsoventral retinal axis. At P0, immunostaining appeared more prominent at the junction of the IPL and RGC layer (arrow in $\boldsymbol{A}^{\prime}$ ). A high degree of nonspecific staining of the lens (asterisk) was seen (B; negative control from Ten-m2 KO mouse), although no staining was present in the retina of control sections. $\boldsymbol{C}, \boldsymbol{D}$, Near to the $0 C$, Ten-m2 expression was observed on axonal tracts traveling to/away from the $0 C$ (arrowheads). No expression of Ten-m2 was seen on cells surrounding the chiasm where RGC axons travel (arrow). Areas that were dorsal to this region, corresponding to areas within the medial preoptic nucleus of the hypothalamus (asterisk), also showed immunoreactivity for Ten-m2. $\boldsymbol{E}-\boldsymbol{H}, \mathbf{U n i f o r m}$ Ten-m2 expression in the dLGN (outlined with dashed line) was observed across the dorsomedial-ventrolateral axis (P7) with in situ hybridization (E). No staining was present in a nearby sense control section $(\boldsymbol{F})$. Immunohistochemistry for Ten-m2 $(\boldsymbol{G})$ shows a similar pattern to mRNA. No staining was present in sections incubated without primary antibody $(\boldsymbol{H})$. $\boldsymbol{I}-\boldsymbol{L}$, The developing $\mathrm{SC}$ also displayed consistent expression across its retinorecipient layers (outlined by arrowheads) as shown by in situ hybridization (I) and immunostaining $(\boldsymbol{K})$. No staining is present in the corresponding control sections $(\boldsymbol{J}, \boldsymbol{L})$. $\boldsymbol{M}-\boldsymbol{P}$, Deep layers of primary visual cortex (V1) expressed Ten-m2 mRNA by P0 (outlined by arrowheads). No staining is present in sense controls $(\boldsymbol{N})$. By P7, immunohistochemistry reveals high expression in layers IV and V (area outlined by arrowheads) ( $\mathbf{0})$. No staining is seen in an adjacent control section (P). Images are representative coronal sections with dorsal to the top. Medial is to the right in all images except $\boldsymbol{C}$ and $\boldsymbol{D}$, where medial is in the center. Dashed outlines in control images indicate areas of interest. Ctx, cortex; hc, hippocampus. Where applicable, the midline is indicated with a dotted line. Scale bars: $200 \mu \mathrm{m}$. 

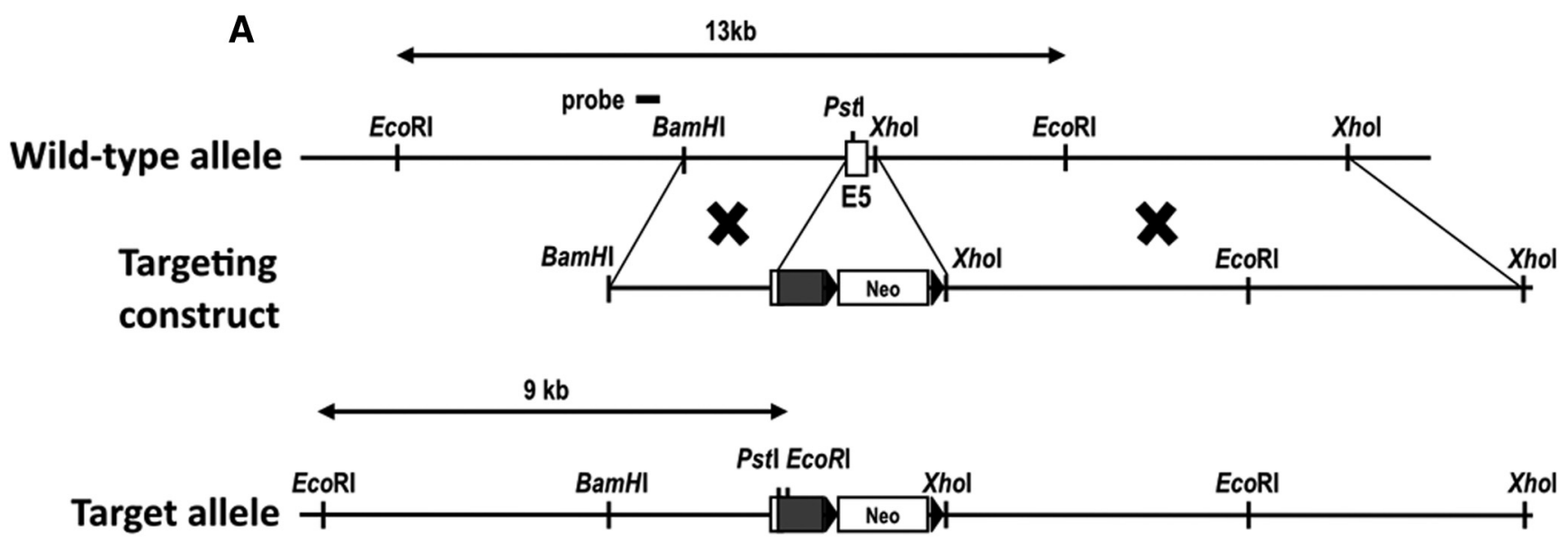

B

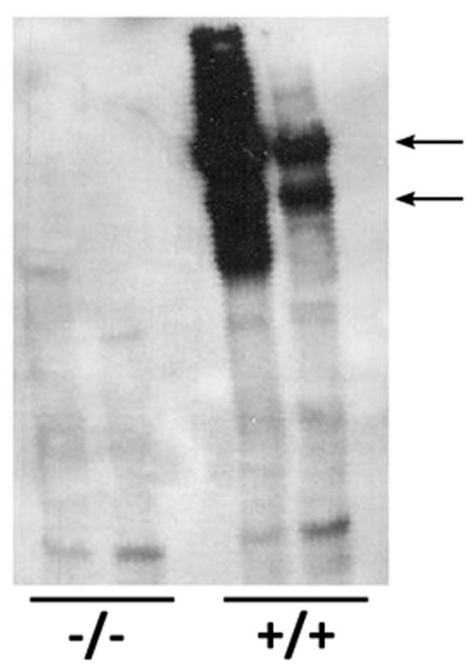

C

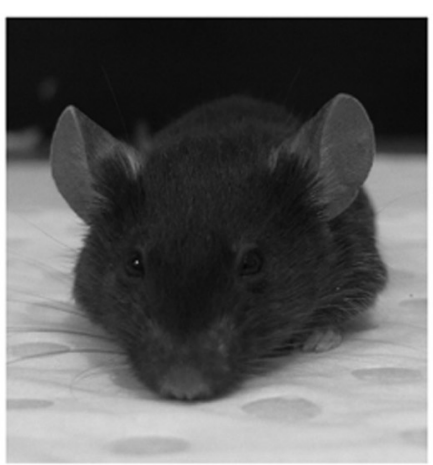

WT

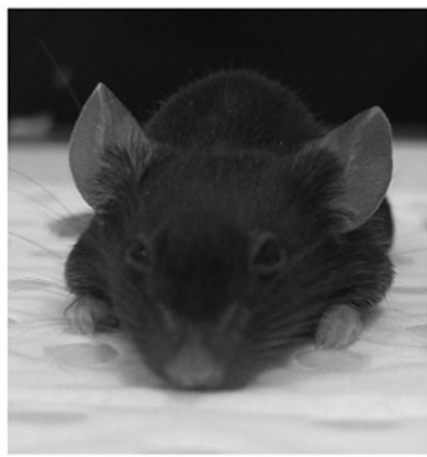

KO

Figure 2. Generation of Ten-m2 K0 mice. A, A schematic illustrating the WT Ten-m2 locus and targeting construct used to generate the Ten-m2 K0 mouse strain. The mutant allele resulting from homologous recombination contained a targeted disruption of exon 5 by insertion of a neomycin-resistance cassette. Detection of the WT (13 kb) and mutant $(9 \mathrm{~kb})$ alleles was performed by Southern blot analysis using a $5^{\prime}$ external probe on EcoRI digests of genomic DNA. B, Confirmation of knockdown of Ten-m2 protein was performed by Western blot using an anti-Ten-m2 antibody specific for its extracellular domain. Two prominent bands were evident at $>400 \mathrm{kDa}$ (arrows), corresponding to the dimerized form of the protein under these conditions (Feng et al., 2002) in lanes loaded with WT $(+/+)$ lysates. These bands are absent in lanes loaded with $\mathrm{KO}(-/-)$ lysates. $C$, Appearance of WT and K0 mice. No noticeable differences in terms of head shape and interocular distance were observed between WT and KO animals.

This relationship was further reflected by a significant decrease in ipsilateral area when considered as a percentage of dLGN area within the rostral part of the nucleus in Ten-m2 KOs (Fig. 3I, sections 6-10; WT, $n=18$; Ten-m2 KO, $n=16$; multivariate ANOVA; s6: $F_{(1,32)}=9.18, p=0.005$; s7: $F_{(1,32)}=35.06, p<$ 0.001 ; s8: $F_{(1,32)}=38.21, p<0.001 ; s 9: F_{(1,32)}=80.80, p<0.001$; s10: $F_{(1,32)}=22.28, p<0.001$; all other sections $\left.p>0.3\right)$. Analysis of horizontal dLGN sections performed in a few cases revealed similar quantitative changes to ipsilateral inputs in Ten-m2 KOs (data not shown). When assessed by threshold analysis (MuirRobinson et al., 2002), the degree of segregation between contralateral and ipsilateral inputs was largely comparable across genotypes (Fig. $3 \mathrm{~J}, \mathrm{~K} ; \mathrm{p}>0.05$ for all threshold values, Student's unpaired $t$ test), with no differences being found, including at rostral levels where the decrease in ipsilateral terminals was seen. This suggests that Ten-m2 acts separately from activitydependent mechanisms to regulate the development of the ipsilateral pathway.

\section{Retinocollicular projections}

Since the retina projects to multiple visual targets, inputs to the $\mathrm{SC}$ were also studied in the same animals. In both genotypes, coronal sections through the SC revealed that contralateral projections completely innervated the superficial retinorecipient layers (i.e., stratum zonale and stratum griseum superficiale) while ipsilateral projections were normally segregated and located deeper within stratum opticum (Fig. $4 A-D$ ). Corresponding to the stereotypic mapping of ipsilateral RGCs (Godement et al., 1984; Hofbauer and Dräger, 1985), these projections were mostly found in a single medial patch in caudal sections of the SC (Fig. $4 A, B)$, while their distribution became extended and more variable along the mediolateral axis of rostral SC (Fig. 4C,D) in both genotypes. It appeared, however, that ipsilateral terminals were reduced in Ten-m2 KOs, compared with WTs. Because of the patchiness of distribution and variability observed in this plane of sectioning, horizontal sections of flattened SCs were analyzed to allow for a better overall topographic and quantitative assessment.

As with coronal sections, ipsilateral inputs were found to be roughly restricted to a rostromedial location in the SC of both genotypes ( $n=9$ SC hemispheres), suggesting that gross topography was largely maintained in KOs (Fig. 4E,F). Notably, however, Ten-m $2 \mathrm{KO}$ mice showed a decrease in the proportion of the 


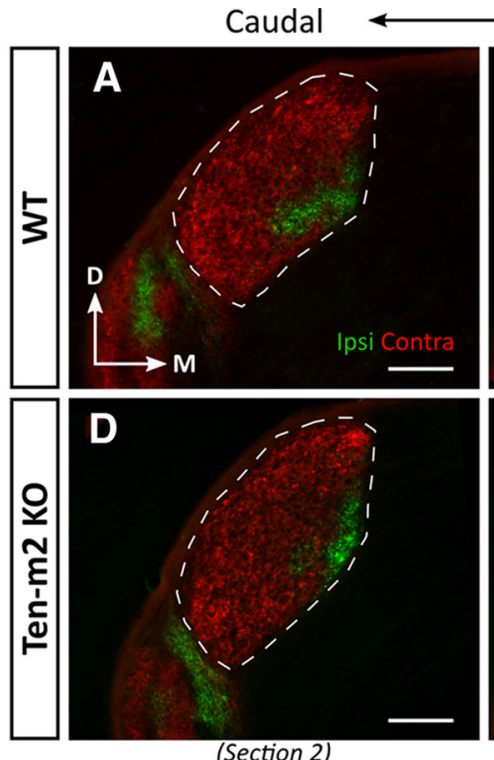

(Section 2)

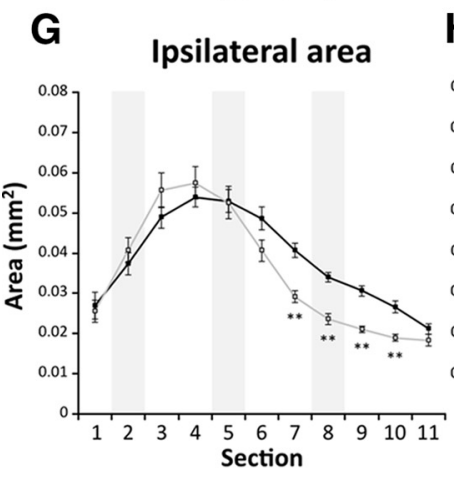

$\mathbf{J}$
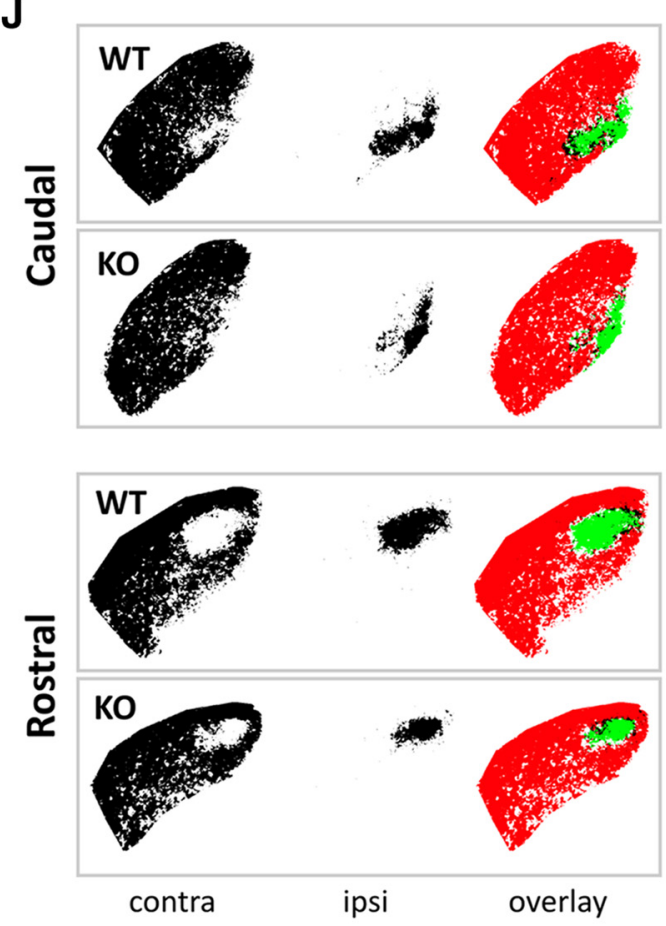

Rostral
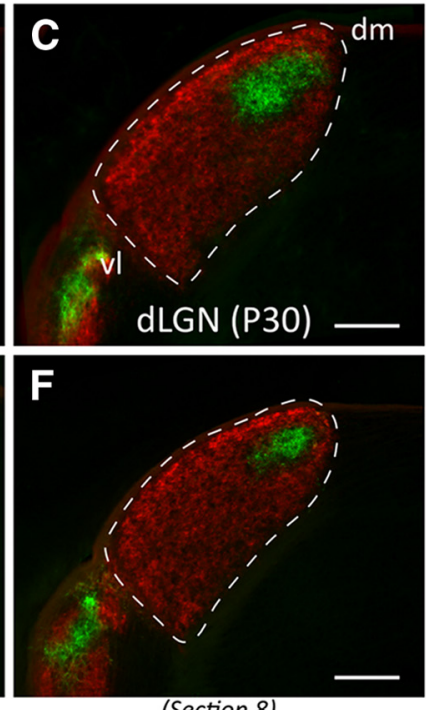

(Section 8)

I
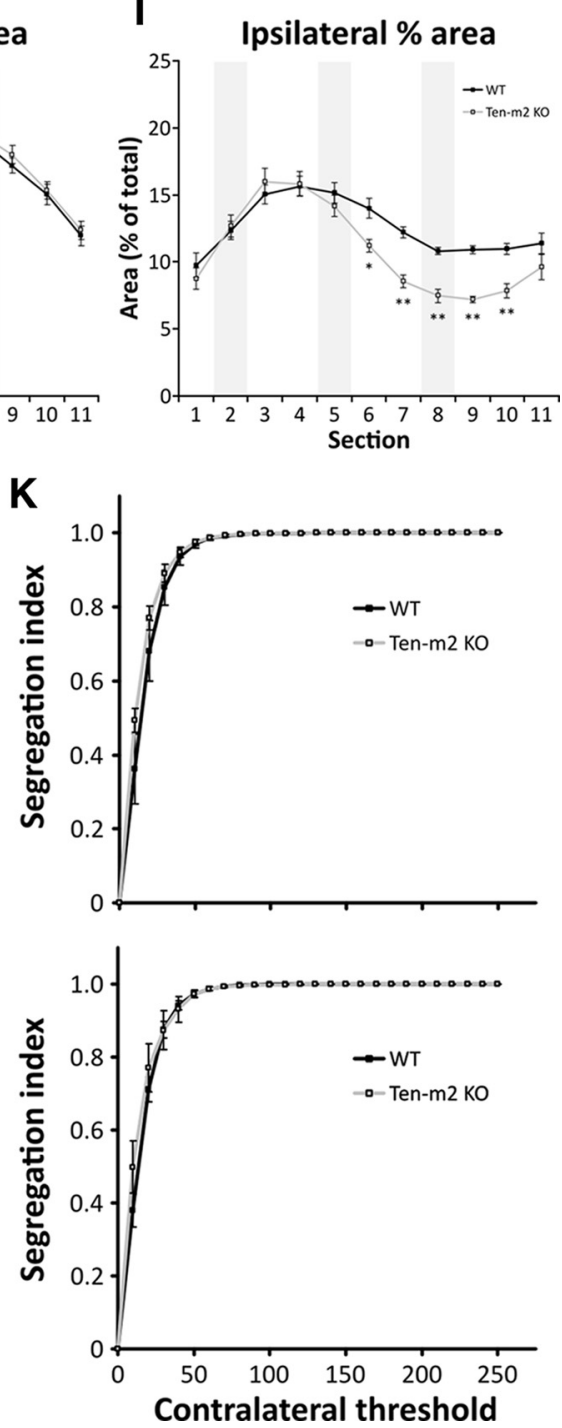

Figure 3. Retinogeniculate projections are distinctly altered in Ten-m2 K0 mice. A series of caudal-to-rostral coronal sections from WT mice at P30 (A-C) demonstrate the stereotypical position of ipsilateral (green) retinal projections terminating within dLGN (outlined), segregated from contralateral (red) inputs, following dual intraocular injections of fluorescently labeled (TB. No difference in segregation or the overall placement of ipsilateral label was found between WT ( $n=18 \mathrm{dLGNs}$ ) and Ten-m2 KO $(n=16 \mathrm{dLGNs} ; \boldsymbol{D}-\boldsymbol{F})$ mice. An overall decrease in ipsilateral projections was found for Ten-m2 K0 mice, which was seen predominantly within rostral dLGN ( $\boldsymbol{F}$ vs $\boldsymbol{C}$. In all images, dorsal is to the top and medial to the right. dm, Dorsomedial; vl, ventrolateral. Scale bar, $200 \mu \mathrm{m}$. $\mathbf{G}-\mathbf{I}$, Quantitative analysis of the caudal-to-rostral series (Figure legend continues.) 
SC occupied by ipsilateral terminals (mean ipsilateral area percentage \pm SEM; WT: $6.28 \pm$ 0.56\%; Ten-m2 KO: $4.22 \pm 0.45 \%$; $p=0.011$, Student's unpaired $t$ test; Fig. $4 I$ ). Heatmaps constructed from thresholded images (Fig. $4 G, H, J$ ) indicated that this reduction occurred mainly within medial regions of SC. Subtraction of these images allowed the location of the changes to be clearly visualized (Fig. 4J). Interestingly, the decrease in retinal ipsilateral input to medial SC corresponds topographically with changes observed in rostral dLGN.

\section{Retrograde WGA-HRP injection shows region-specific changes to the ipsilateral RGC population in Ten-m $2 \mathrm{KO}$ retina}

Injections of WGA-HRP were made into the dLGN of WT and Ten-m 2 KO mice to gain a better understanding of the changes to ipsilateral projections described above. Comprehensive filling of the dLGN with WGA-HRP allowed retrograde labeling of retinogeniculate projections from both contralateral and ipsilateral retinae. Complete fills of the dLGN in the absence of spread to the other hemisphere were confirmed by examination of sections stained for WGA-HRP through the extent of the nucleus. In WT mice, this resulted in the labeling of RGCs in the ipsilateral retina that were restricted to the VTC (Fig. 5A). In WT mice, the number, and area occupied by, ipsilaterally labeled cells was largely comparable to data found in the literature (Dräger and Olsen, 1980) (Fig. 5C,D; mean number \pm SEM: $1016 \pm 93$ cells; mean area \pm SEM: $\left.2.29 \pm 0.12 \mathrm{~mm}^{2}, n=5\right)$. In Ten-m2 KO mice the most striking change was a significant decrease in the size of the region occupied by retrogradely labeled ipsilaterally projecting RGCs (Fig. $5 B, D$; mean area \pm SEM: $1.43 \pm 0.16 \mathrm{~mm}^{2}, n=4$; $p=0.003$, Student's unpaired $t$ test). Since the density of labeled cells in VT retina was not significantly different between genotypes (Fig. 5E; mean ipsilateral cell density \pm SEM; WT: $444 \pm 34$ cells $/ \mathrm{mm}^{2}$; Ten-m2 KO: $436 \pm 51$ cells $/ \mathrm{mm}^{2} ; p=0.896$, Student's unpaired $t$ test), this correlated with a significant decrease in the number of labeled cells in the VTC in Ten-m2 KOs (Fig. $5 C$; mean number \pm SEM: $606 \pm 51$ cells; $p=0.008$, Student's unpaired $t$ test) compared with WT. This decrease is in agreement with the anterograde CTB labeling studies. No difference was found in total retinal area between genotypes (mean area \pm SEM; WT: $12.41 \pm 0.31 \mathrm{~mm}^{2}, n=10$ retinae; Ten-m2 KO: $12.16 \pm$ $0.25 \mathrm{~mm}^{2}, n=8 ; p=0.551$, Student's unpaired $t$ test). The notable reduction in the area of retrogradely labeled ipsilaterally projecting RGCs in Ten-m2 KOs was also observed when considered as a percentage of total retinal area (mean VT area percentage \pm SEM; WT: $18.30 \pm 1.14 \%$; Ten-m2 KO: $12.27 \pm 1.04 \%$; $p=0.004$, Student's unpaired $t$ test).

\footnotetext{
$\leftarrow$

(Figure legend continued.) (sections 1-11) through the dLGN revealed that absolute area occupied by ipsilateral terminals $(\boldsymbol{G})$ was significantly reduced in a number of sections (7-10) through rostral Ten-m2 KO dLGN (light gray), compared with WT (black). These changes occurred in the absence of significant differences in total dLGN size $(\boldsymbol{H})$, and were similarly observed when ipsilateral area was considered as a percentage of total dLGN area (I). Representative images shown in $\boldsymbol{A}-\boldsymbol{E}$ correspond to sections 2,5 , and 8 along the caudal-torostral series in $\mathbf{G}-\mathbf{I}$ (shaded areas). ${ }^{*} p<0.05,{ }^{* *} p<0.005$, in comparisons to WT; multivariate ANOVA. J, Segregation of contralateral (left, red) and ipsilateral (middle, green) inputs to the dLGN was assessed in caudal (top) and rostral (bottom) dLGN using a threshold analysis. Overlapping contralateral and ipsilateral regions are indicated as black in image overlays (right). $\boldsymbol{K}$, At each contralateral threshold value, segregation indices were not significantly different ( $p>0.05$ ) between WT (black) and Ten-m2 K0s (light gray) for either caudal (top) or rostral (bottom) locations, indicating comparable segregation of inputs in these animals.
}

Intriguingly, the loss of ipsilaterally projecting cells appeared to be consistently localized to a specific subregion of the VTC in all Ten-m2 KOs: labeled cells appeared to be largely conserved in temporal regions but were strikingly absent from more ventral retina (Fig. $5 B$ ). To analyze these changes more thoroughly in terms of their retinal location and dimension, we calculated the distance of the arc separating the nasal fiducial cut and the temporal and ventral extremes of the ipsilateral label, and expressed this as a fraction of retinal circumference (Fig. $5 F, G$ ). We found that while the ratio of the arc measuring the distance to the temporal edge of the ipsilateral label did not vary between the groups (Fig. $5 G$; temporal arc ratio, mean \pm SEM; WT: $0.44 \pm 0.02, n=$ 5, KO: $0.46 \pm 0.02, n=4 ; p=0.388$, Student's unpaired $t$ test) the distance to its ventral border was significantly greater in KOs (Fig. $5 G$; ventral arc ratio, mean \pm SEM; WT: $0.22 \pm 0.02 ; \mathrm{KO}: 0.32 \pm$ $0.03, p=0.036$, Student's unpaired $t$ test). Consistent with this change, the ratio of the arc encompassed by the labeled region to the total retinal circumference was reduced in KOs (ipsilateral arc ratio, mean \pm SEM; WT: $0.34 \pm 0.012, n=5$; KO: $0.22 \pm 0.017$, $n=4 ; p=0.001$, Student's unpaired $t$ test). We also investigated the possibility of changes across the orthogonal, radial axis of the retina. Although less obvious than the circumferential changes, we found a significant decrease in the fraction of the retinal radius encompassed by the ipsilaterally labeled RGCs in Ten-m2 KOs (Fig. 5G; ipsilateral radial ratio, mean \pm SEM; WT: $0.35 \pm 0.005$; KO: $0.26 \pm 0.011 ; p=0.002$, Student's unpaired $t$ test). Thus, the decrease in the number of ipsilaterally projecting RGCs in Ten-m2 KOs is comprised of changes across both the radial and circumferential dimensions of the retina. The primary and most striking change is the loss of ipsilaterally projecting RGCs from ventral retina, evidenced by the marked increase in the ventral arc ratio, in the absence of a change in the temporal arc ratio. The decrease in the ipsilateral radial ratio, however, shows that more subtle changes are also present more dorsally, within the temporal region of the VTC.

Corresponding changes were also observed in contralateral retinae (Fig. 6). In WTs, closely packed labeled cells were observed across most of the contralateral retina, with the exception of the VTC region, where labeled cells were present at a lower density. These cells presumably correspond to late-born contralateral RGCs that have been previously described to occupy this region (Dräger, 1985; Guillery et al., 1995; Williams et al., 2006). In Ten-m 2 KO mice, however, contralaterally labeled cells were found to have expanded ventrally into this normally labelsparse region, resulting in a significant decrease in the area of the VTC in Ten-m2 KOs (Fig. 6A-C; mean contralateral VT area \pm SEM; WT: $1.27 \pm 0.08 \mathrm{~mm}^{2}, n=5$; Ten-m2 KO: $0.81 \pm 0.12$ $\mathrm{mm}^{2}, n=4 ; p=0.019$, Student's unpaired $t$ test). Outside the VT region, the labeling of contralateral cells appeared largely similar between genotypes with regards to the density (Fig. 6D; mean cell density \pm SEM; WT: $2608 \pm 92$ cells $/ \mathrm{mm}^{2}$; Ten-m2 KO: $2808 \pm$ 145 cells $/ \mathrm{mm}^{2} ; p=0.264$, Student's unpaired $t$ test) and estimated number of cells (mean cell number \pm SEM; WT: 28,633 \pm 1659 cells; Ten-m2 KO: $32,363 \pm 2551$ cells; $p=0.243$ ).

These data provide strong evidence of a reduction in ipsilateral projections that arise predominantly, but not exclusively, from the ventral region of the retina in Ten-m2 KOs. Further, these changes correspond topographically with the deficits observed in rostral dLGN (Reese and Jeffery, 1983), and medial SC (Reese, 1986) of KO mice. It therefore appears that the neuroanatomical phenotype of Ten-m2 KO mice arises due to a perturbation in specification of laterality in the retina and/or decussation at the OC. 

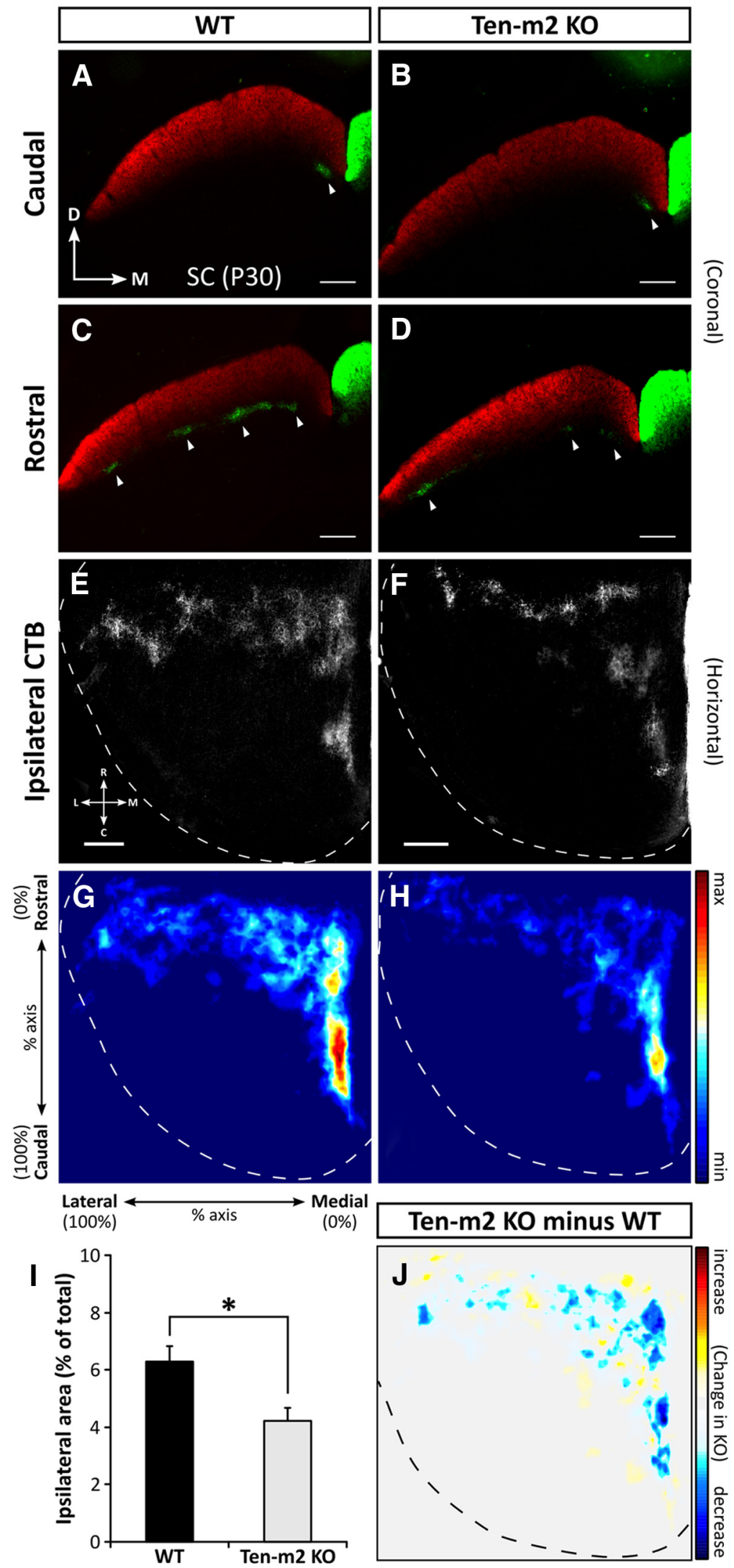

Figure 4. Alterations to retinocollicular projections are also observed in Ten-m2 $\mathrm{K} 0$ mice. Following dual intraocular injections of fluorescently labeled $\mathrm{CTB}$, coronal sections $(\boldsymbol{A}-\boldsymbol{D})$ through the $\mathrm{SC}$ show contralateral retinal projections (red) occupying the entire superficial, retinorecipient layers of the SC. In the same SC hemisphere, ipsilateral terminals (green) are segregated and situated deeper within stratum opticum (arrowheads) in both WT and Ten-m2 K0 mice. In WT mice, ipsilateral inputs were restricted to medial locations in caudal SC $(\boldsymbol{A})$, while they were variably distributed in clusters along the mediolateral axis of rostral
Evidence for Ten-m 2 in the regulation of ipsilateral fate downstream of Zic2 expression

The reduction in the size of the ipsilateral projections in Ten-m2 KOs is somewhat similar to the phenotype observed in mice that have had known determinants of the ipsilateral pathway altered (Herrera et al., 2003; Williams et al., 2003; Fabre et al., 2010; Rebsam et al., 2012). A notable distinction, however, is that ventral retina was predominantly affected in Ten-m2 KOs. Such a phenotype could potentially arise due to regionally localized cell death. A more likely possibility is that Ten-m2 might play a role in the molecular pathway, which defines cell fate with respect to laterality. To help distinguish between these possibilities, and define potential molecular interactions of Ten-m2, we examined the expression of a chief determinant of laterality fate, Zic2, at E16.5 in WT and Ten-m2 KO retina. This date was chosen as it corresponds to the peak of Zic2 expression (Herrera et al., 2003), but is well before the peak of programmed cell death, which occurs postnatally (Péquignot et al., 2003).

Immunostaining of whole-mount retinae from E16.5 embryos revealed Zic2positive cells in the VT region, as well as a high-density region of proliferating cells in the ciliary marginal zone (CMZ; Herrera et al., 2003). The location, area, and number of Zic2-positive cells in VT retina showed no major differences between WT and Ten-m2 KO mice (Fig. 7A-C). Most notably, there was no evidence of diminished Zic2 staining in the ventral retina where ipsilateral projections were absent

SC (C). While no difference was seen caudally in $\mathrm{KOs}(\boldsymbol{B})$, there was evidence that ipsilateral inputs were decreased in Ten-m2 KOS (D) within rostral SC sections. For images in $\boldsymbol{A}-\boldsymbol{D}$, dorsal (D) is to the top and medial (M) to the right. Scale bar, $200 \mu \mathrm{m}$. $E, F$, Overall distribution of ipsilateral label was best demonstrated in horizontal sections through the SC (one hemisphere outlined), occupying a stereotypical rostromedial location in WTs $(\boldsymbol{E})$. The proportion of the SC occupied by ipsilateral terminals was decreased in Ten-m2 K0s, compared with WTs $(\boldsymbol{F})$. $\boldsymbol{G}, \boldsymbol{H}$, Distribution for each genotype is shown by composite heatmaps of ipsilateral label from each animal $(n=9$ hemispheres for each genotype). Regions in red indicate high intensity of label, while blue regions indicate low levels of label. In Ten-m2 K0 mice, ipsilateral label area was significantly reduced $(\boldsymbol{I})$, most notably in medial $\mathrm{SC}(\boldsymbol{H})$. These changes are highlighted when WT signal is subtracted from KO signal (J), with blue regions showing where label is decreased in $\mathrm{K} 0$, with respect to WT. Images in $\boldsymbol{E}$-J are in the horizontal plane with rostral to the top, and medial to the right. Scale bar, $250 \mu \mathrm{m}$. For heatmaps, signal is plotted along the percentage of the rostrocaudal and mediolateral axes of the SC; color scales are shown to the right. ${ }^{*} p<0.05$, Student's unpaired $t$ test. 
in these KOs (Fig. $7 A, B$ ). Similarly, realtime RT-PCR revealed no significant differences in Zic2 (or related Zic1) mRNA expression between WT and Ten-m2 KO retina (relative mRNA expression \pm SEM, Ten-m2 KO vs WT; Zic1: $1.33 \pm 0.08$-fold; Zic2: $0.85 \pm 0.14$-fold; $p>0.05$; fixed pairwise reallocation randomization test).

The unaltered levels of Zic2 expression in $\mathrm{KO}$ mice do not exclude the possibility that Ten-m2 may act downstream of this transcription factor to regulate expression, or directly interact with it, or its target genes. To test whether Ten-m2 functions in the retina to regulate expression of genes that are driven by Zic2, we looked for differences in the expression of EphB1, which is known to facilitate repulsion of RGC axons at the chiasm via interaction with its EphrinB2 ligand (Williams et al., 2003; Petros et al., 2009). As previously reported, in situ hybridization revealed WT EphB1 expression at E16.5 was present in the peripheral portion of temporal (Fig. 7D) to ventral (Fig. $7 G$ ) retina, corresponding to the VTC (Williams et al., 2003). In Ten-m2 KOs, although EphB1 expression appeared unaltered in temporal regions (Fig. 7E), a region-specific reduction was apparent in peripheral, ventral retina (Fig. $7 H$ ). Semiquantitative analysis of coronal sections taken from a comparable temporal level showed no significant differences between genotypes in the area or density of EphB1 signal (Fig. 7J; temporal EphB1 pixel area \pm SEM; WT: $1323.9 \pm 49.9 \mu \mathrm{m}^{2}$; Ten-m2 KO: $1148.0 \pm 68.3 \mu \mathrm{m}^{2} ; p=0.087$; EphB1 pixel density \pm SEM; WT: $3.27 \pm$ 0.29 units; Ten-m2 KO: $3.13 \pm 0.17$ units; $p=0.693$; Student's unpaired $t$ test, $n=4$ for each genotype). A similar comparison of ventral retina, however, confirmed the specific reduction in this region, with both area and density of EphB1 signal significantly decreased in Ten-m2 KO retina (Fig. 7J,K; ventral EphB1 pixel area \pm SEM; WT: $500.1 \pm 54.4 \mu \mathrm{m}^{2}$; Ten-m2 KO: $295.4 \pm 27.9 \mu \mathrm{m}^{2} ; p=0.024 ; \mathrm{EphB1}$ pixel density \pm SEM; WT: $2.79 \pm 0.28$ units; Ten-m2 KO: $1.65 \pm 0.13$ units; $p=$ 0.018; Student's unpaired $t$ test, $n=4$ for each genotype). These data indicate a requirement of Ten-m2 for EphB1 expression in ventral retina. The use of coronal sections in this analysis (as opposed to the whole mounts used for retrograde tracing studies) made it difficult to assess a potential change across the radial axis of the retina in this material. Nevertheless, together with the retrograde tracing studies above, these data raise the possibility that the primary neuroanatomical defect in the retinofugal pathway of Ten-m $2 \mathrm{KO}$ mice arises as a consequence of reduced EphB1 expression, which in turn causes impaired repulsion of ipsilateral axons predominantly arising from ventral retina.

C

$\mathbf{F}$
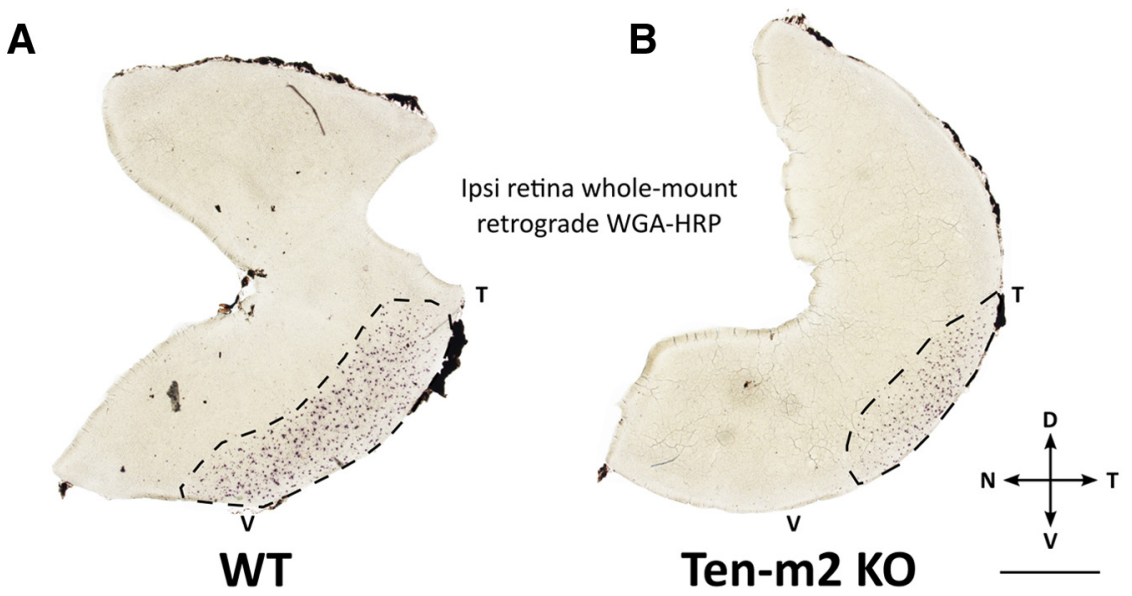

D

E
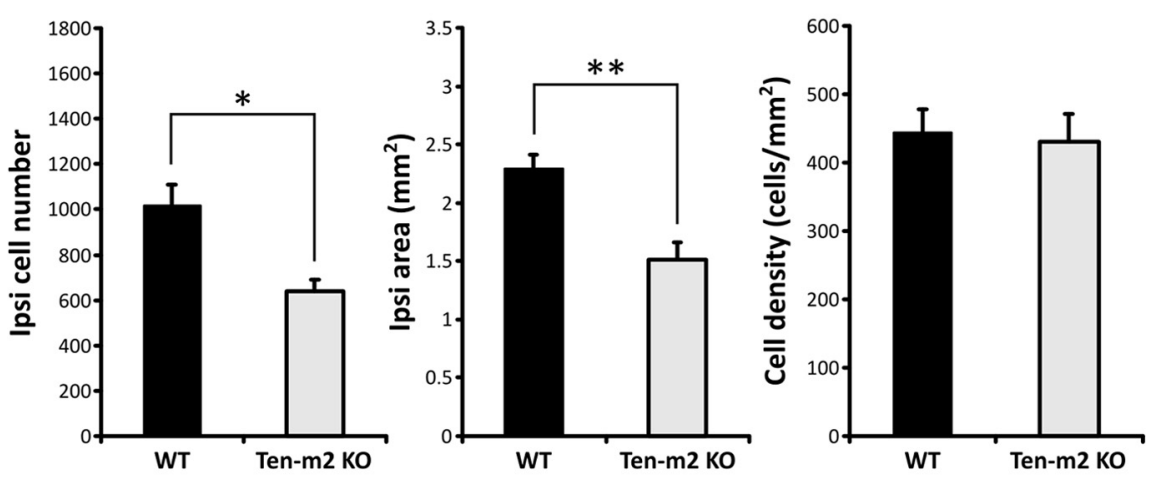

G



Figure 5. Retrograde tracing of ipsilateral retinogeniculate projections reveals distinct changes in Ten-m2 K0 mice. Ipsilateral retinogeniculate projections were retrogradely labeled with stereotaxic WGA-HRP injections into the right dLGN. $A, B$, Labeled cells (dark spots; area outlined by dotted line) were visualized in whole mounts of ipsilateral retinae, with cells in WT $(n=5)$ found in a stereotypical position in VT retina $(\boldsymbol{A})$. Both the number $(\boldsymbol{C})$ and area occupied $(\boldsymbol{D})$ by ipsilateral cells were significantly reduced in Ten-m2 K0 retina $(n=4)(\boldsymbol{B})$. No differences were found in cell density $(\boldsymbol{E})$ between genotypes. $\boldsymbol{F}$, Schematic diagram outlining analysis of changes in the population of ipsilaterally labeled cells. Using the nasal fiducial cut, temporal and ventral arc length was expressed as a ratio of retinal circumference. The width of the ipsilateral label was expressed as a ratio of length across the radius of the retina. G, Ventral arc ratio was significantly increased in Ten- $\mathrm{m} 2 \mathrm{KO}$ retina, indicating the loss of ipsilaterally projecting cells from ventral retina, while no change in temporal arc ratio was found. Furthermore, a reduction in the ipsilateral radial ratio in Ten-m2 KOs indicated that changes also occur along the radial dimension of temporal retina. Dorsal is to the top, temporal to the right in all images. Scale bar, $1 \mathrm{~mm} .{ }^{*} p<0.05,{ }^{* *} p<0.01$, in comparisons to WT; Student's unpaired $t$ test.

Increased contralateral projections from ventral retina in Ten-m2 KOs have normal topography

Previous studies have demonstrated that in EphB1 KOs, there is a misrouting of axons at the chiasm to produce an expanded contralateral projection, which maps in the manner of ipsilateral axons within the dLGN (Rebsam et al., 2009). To build on 
A
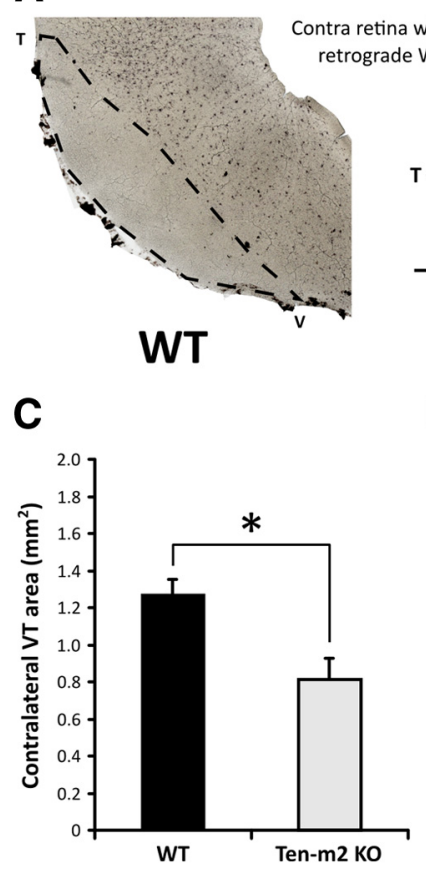

D

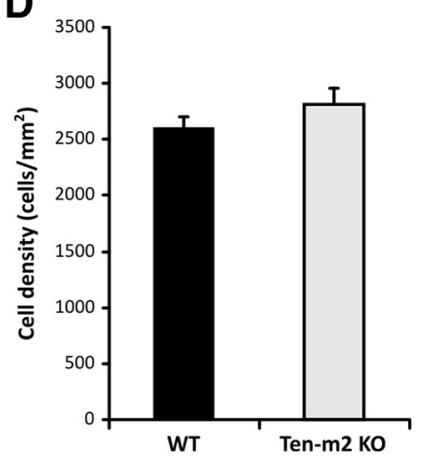

Figure 6. Ten-m2 KO mice display corresponding changes to contralateral retinogeniculate projections from VT retina. Retrograde tracing of contralateral retinogeniculate projections was achieved using stereotaxic WGA-HRP injections into dLGN. $\boldsymbol{A}, \boldsymbol{B}$, The VT quadrants of whole-mounted retinae, contralateral to the injections are shown. Labeled cells fill the bulk of this region, however, the VTC region, corresponding to the origin of ipsilateral projections (outlined), was largely devoid of labeled cells. The area of the sparsely labeled contralateral VT region ( $C$ was significantly reduced in Ten-m $2 \mathrm{KO}$ mice, due to an expansion of retrogradely labeled contralaterally projecting cells into the ventral part of the VTC $(\boldsymbol{B})$. The density of labeled cells within the central region of the retina was not significantly different across genotypes $(\boldsymbol{D})$. Scale bar, $1 \mathrm{~mm}$. Dorsal (D) is to the top, temporal (T) to the left, nasal $(\mathrm{N})$ to the right, ventral $(\mathrm{V})$ to the bottom. ${ }^{*} p<0.05$, Student's unpaired $t$ test.

our findings from retrograde tracing and Zic2/EphB1 expression experiments, therefore, and to assess topography in more detail, focal DiI injections were made to target RGCs in the ventral half of the VTC in WT $(n=5)$ and Ten-m2 KO $(n=3)$ mice at P13. Comparable injections (size and location) resulted in the labeling of a small population of RGCs that project to contralateral and ipsilateral dLGN (Fig. 8A,B). Interestingly, no overall difference in topography was seen between the genotypes. Consistent with the normal visuotopic alignment of projections to the dLGN, contralateral label was located in a single termination zone (TZ) at the dorsomedial dLGN pole, while ipsilateral TZs were displaced ventrolaterally in both WT and Ten-m2 KO mice. In Ten-m2 KOs, however, the extent of ipsilateral TZs was smaller, while label in the contralateral dLGN appeared expanded, compared with WTs and in relation to the degree of label on the ipsilateral side. Quantification confirmed that the ratio of ipsilateral-tocontralateral label seen in the dLGN was consistently lower in Ten-m2 KO animals (Fig. $8 C$; ratio of ipsi-to-contra label \pm SEM; WT: $1.35 \pm 0.38$; Ten-m2 KO: $0.57 \pm 0.12 ; U<0.001$, $p=0.036$, Mann-Whitney $U$ test). The $>1$ ratio in WTs likely reflects the normal amplified representation of ipsilateral projections that is present in the dLGN (LaVail et al., 1978). Overall, these data argue against misrouting of ipsilateral RGC axons at the chiasm as occurs in EphB1 mutants (Rebsam et al., 2009), but rather a switching of laterality fate further up- stream, resulting in coordinated changes in both ipsilateral guidance at the midline and mapping within the target. This, together with the reduced expression of EphB1, suggests that Ten-m 2 acts to promote the expression of a coordinated, ipsilateral-specific suite of genes in the ventral VTC, and may also repress a contralateral guidance program in these cells. These changes occur downstream (or possibly independently) of Zic2 expression.

\section{Ipsilaterally driven activity within V1 is altered in Ten-m2 KO mice}

The unique neuroanatomical phenotype we have discovered in the Ten-m2 KO mice suggests a functional impairment of binocular vision, particularly in regions involved in processing the dorsal visual field. Labeling of geniculocortical projections revealed that the overall topography of projections from the dLGN to V1 is largely normal in Ten-m2 KOs (data not shown), raising the possibility that the specific reduction in ipsilateral input from ventral retina is conveyed topographically to the cortex of these mice. As a means of first determining whether the degree of ipsilateral drive to V1 is altered in Ten-m2 KOs, immunostaining for the immediate early gene, c-fos, was performed in acute monocularly inactivated animals (Dragunow and Robertson, 1988). Under these conditions, induction of c-fos expression within V1 corresponds to neuronal activity that has been driven by inputs arising exclusively from the active eye. In WT mice, c-fos label on the side ipsilateral to the active eye was restricted to the lateral-most region of $\mathrm{V} 1$, corresponding to the binocular zone (Fig. 9A). While the ipsilateral signal in Ten-m2 KOs was confined to lateral V1 (Fig. 9B), as in WTs, a slight, but significant reduction was observed compared with WT values, for both area (mean ipsilateral label area \pm SEM, WT: $1.46 \pm 0.08 \mathrm{~mm}^{2}, n=4$; Ten-m2 KO: $1.14 \pm 0.08 \mathrm{~mm}^{2}, n=6 ; p=0.027$, Student's unpaired $t$ test) and mediolateral width (mean ipsilateral label width \pm SEM, WT: $0.75 \pm 0.04 \mathrm{~mm}^{2}, n=4$; Ten-m2 KO: $0.58 \pm$ $0.03 \mathrm{~mm}^{2}, n=6 ; p=0.015$, Student's unpaired $t$ test).

While the overall shape and location of V1 was similar for both genotypes, a slight, albeit nonsignificant, reduction in total $\mathrm{V} 1$ area was also found for Ten-m2 KOs (Fig. 9C; mean total V1 area \pm SEM, WT: $3.67 \pm 0.15 \mathrm{~mm}^{2}, n=4$; Ten-m2 KO: $3.32 \pm$ $0.16 \mathrm{~mm}^{2}, n=6 ; p=0.179$, Student's unpaired $t$ test). To take this possible slight difference into consideration, measurements of ipsilateral activation were normalized to V1 size. Both the percentage of total V1 area occupied by the ipsilaterally driven region (Fig. 9D; mean ipsilateral area percentage \pm SEM, WT: $39.9 \pm 1.5 \%, n=4$; Ten-m2 KO: $34.0 \pm 1.0 \%, n=6, p=0.010$, Student's unpaired $t$ test), and mediolateral width (Fig. $9 E$; mean ipsilateral width percentage \pm SEM, WT: $42.5 \pm 2.1 \%, n=4$; Ten-m2 KO: $34.7 \pm 1.3 \%, n=6 ; p=0.010$, Student's unpaired $t$ test) remained significantly less than in WTs. Additionally, no significant difference in density of ipsilateral label was seen between WTs and Ten-m2 KOs (Fig. 9F; mean density \pm SEM, WT: $0.036 \pm 0.006$ units, $n=4$; Ten-m2 KO: $0.039 \pm 0.004$ units, $n=$ $6 ; p=0.677$, Student's unpaired $t$ test).

Composite heatmaps further suggested that while ipsilateral c-fos immunolabeled in Ten-m2 KOs was still present above background levels within the caudal end of lateral V1, this appeared lower when compared with WTs $(n=4$ for each genotype; Fig. 9G,H). Subtraction of Ten-m2 KO from WT signal revealed the greatest differences between genotypes within this caudal region (Fig. 9I). On the contralateral side, no notable differences were found, with a uniform distribution of c-fos-positive cells being observed across V1 for both genotypes (data 
not shown). Together these results indicate an overall reduction in the degree of ipsilateral input, in Ten-m $2 \mathrm{KO}$ mice. This is most marked in the caudal portion of binocular $\mathrm{V} 1$, corresponding to the representation of ventral retina.

\section{Contribution of ipsilateral inputs to binocular $\mathrm{V} 1$ responses is altered in Ten-m2 KO mice}

Our c-fos data revealed significant differences between WT and Ten-m2 KO mice in ipsilateral drive to V1. To further characterize the contribution of the ipsilateral eye to visual drive, VEP recordings were performed on anesthetized animals from both genotypes. For both WT and Ten-m2 $\mathrm{KO}$ mice, recordings made from lateral V1 ( $\geq 67 \%$ of mediolateral axis; this is within the binocular zone for both WT and KOs as determined by c-fos staining) showed maximal binocular responses when visual stimuli were presented around the vertical meridian $\left(-7.5\right.$ to $\left.22.5^{\circ}\right)$ of the contralateral visual field (Fig. 10A). Moving the recording electrode toward the lateral border of $\mathrm{V} 1 \mathrm{re}$ sulted in a shift in maximal binocular responses toward the vertical meridian for both genotypes, consistent with the normal pattern of visual topography (Dräger, 1975; Wagor et al., 1980; Wang and Burkhalter, 2007). For technical reasons it was not possible to access caudal V1, which receives input from ventral retina where the most dramatic changes were seen. Our recordings therefore addressed the impact of the partial switch in laterality fate in the temporal segment of the VTC.

When the maximal responses from multiple recording sites were averaged and compared across genotypes, several observations were made. Recorded VEP waveforms consisted of a major positive component that was immediately followed by a major negative component, and this overall shape was largely consistent across genotypes (Fig. 10). The relationship between binocular and monocular activation, however, differed between the two groups. In WT mice, the magnitude of responses with regards to peak, trough, and peak-to-trough were greatest upon binocular $>$ contralateral $>$ ipsilateral presentations of visual stimuli (Fig. 10D,F).

For KOs, VEP responses exhibited different properties depending on the location of maximal activation within the visual field. For recordings exhibiting maximal responses from stimuli presentations at the most central region of the visual field tested $\left(-7.5\right.$ to $\left.7.5^{\circ}\right)$, no significant differences across binocular/monocular presentations were found between genotypes for all parameters assessed (Fig. 10B and data not shown).
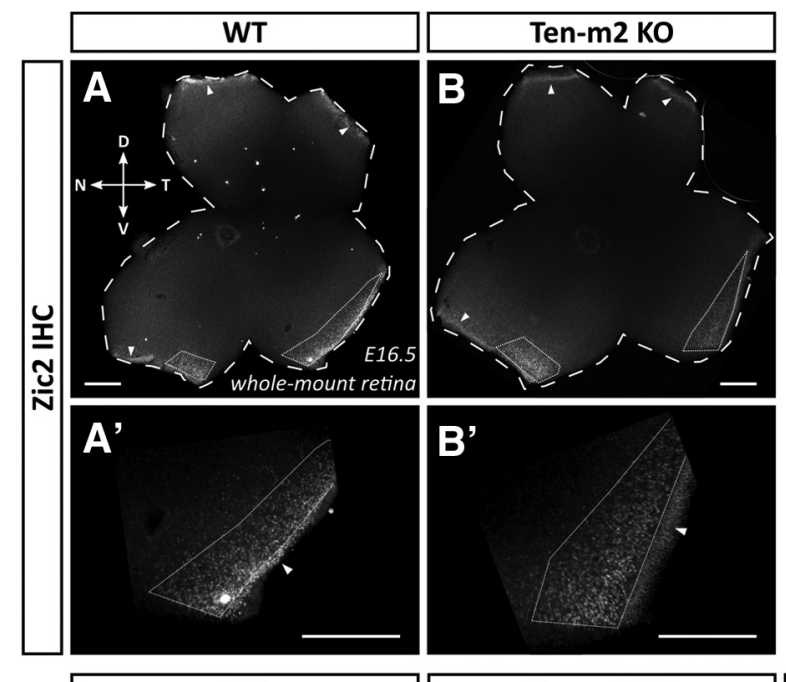

C Zic2 expression
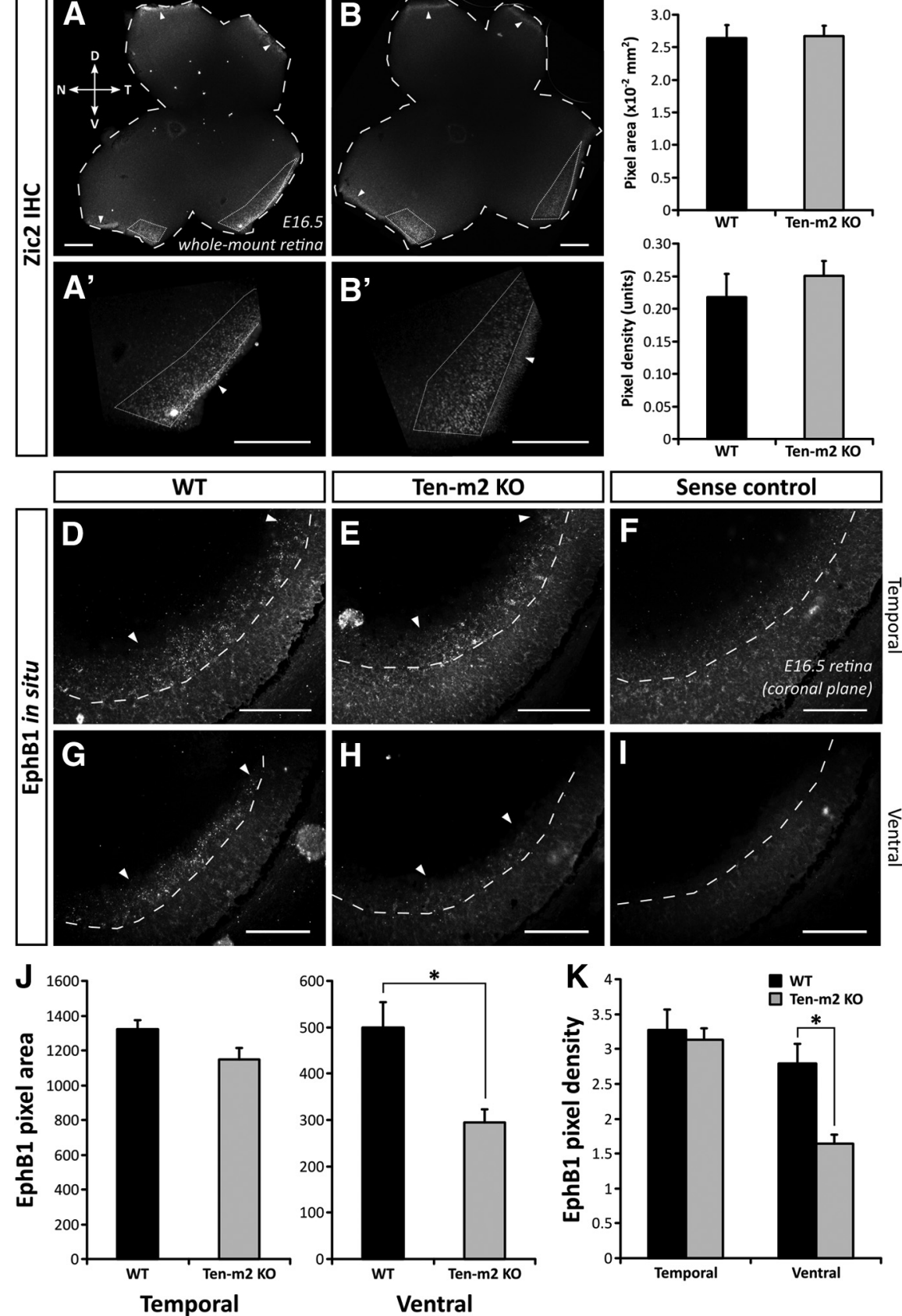

Figure 7. EphB1 expression is altered in the absence of changes to Zic2 expression in Ten-m2 KO retina. $\boldsymbol{A}, \boldsymbol{B}$, Immunohistochemistry against Zic2 on E16.5 whole-mount retinae (bold outline) revealed normal expression in WT and Ten-m2 K0 mice, being restricted to VT retina (dotted outline) in both genotypes. Higher magnification images are shown in $\boldsymbol{A}^{\prime}$ and $\boldsymbol{B}^{\prime}$. Normal expression in the CMZ is also seen in both genotypes (arrowheads). C, Quantitative analysis yielded no differences in area or density of Zic2-positive label. D-I, In situ hybridization for EphB1 in E16.5 retinal sections revealed expression which was restricted to VT retina. In comparable coronal sections through temporal retina, no difference in EphB1 expression was found between WT (D) and Ten-m2 KO $(\boldsymbol{E})$ mice. In contrast, EphB1 expression appeared lower in more peripheral, ventral regions in Ten-m2 K0s $(\boldsymbol{H})$, compared with WTs $(\boldsymbol{G})$. Sense controls are provided for WTs $(\boldsymbol{F})$ and KOs $(\boldsymbol{I})$, respectively. The extent of EphB1 expression within the ganglion cell layer (dashed outline) is indicated by arrowheads. A semiquantitative analysis of EphB1 expression revealed a significant difference in pixel area $(\boldsymbol{J})$ and density $(\boldsymbol{K})$ in ventral, but not temporal, retina of Ten-m2 K0s. ${ }^{*} p<0.05$, Student's unpaired $t$ test. Dorsal is to the top, temporal to the right in $\boldsymbol{A}-\boldsymbol{B}^{\prime}$. Scale bars: (in $\boldsymbol{A}-\boldsymbol{B}^{\prime}$ ) $200 \mu \mathrm{m}$; (in $\boldsymbol{D}-\boldsymbol{I}$ ) $100 \mu \mathrm{m}$.

This was not the case, however, for VEPs elicited maximally from stimuli presented at the immediately adjacent (pericentral) region within the binocular zone of the visual field $\left(7.5-22.5^{\circ}\right)$ (Fig. 10C-F). Here, binocular responses in Ten-m2 KOs $(n=6)$ were not consistently larger than contralateral responses from 

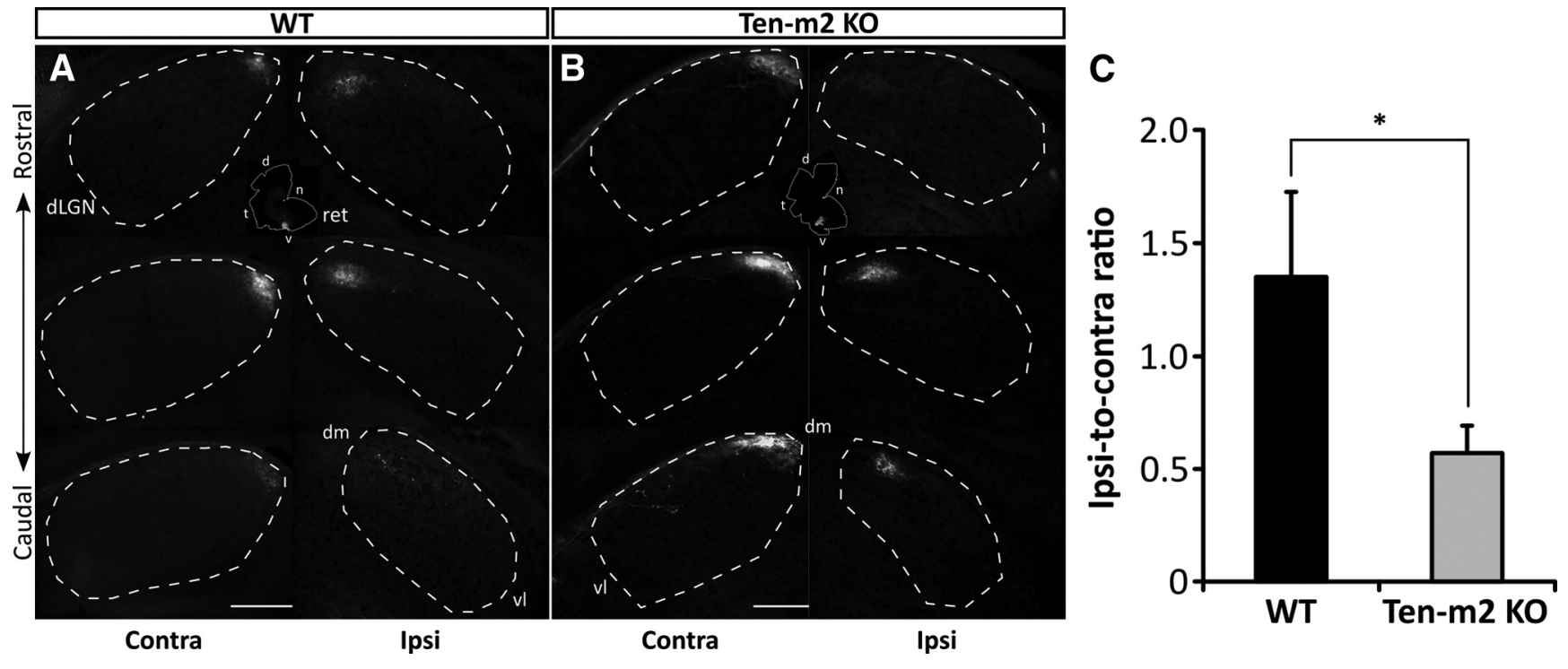

Figure 8. Reduced ipsilateral and expanded contralateral projections from ventral retina map with normal topography in Ten-m2 K0 mice. Focal injections of Dil were made into ventral retina (dotted outline; inset) of WT (A) and Ten-m2 KO (B) mice at P13. In both WT and Ten-m2 KOs, contralateral TZs were located within the dorsomedial pole of dLGN (dashed line), while ipsilateral TZs were displaced ventrolaterally. In Ten-m2 KOs, ipsilateral label was consistently reduced, while label appeared expanded in the contralateral dLGN. Despite this expansion, only a single patch of contralateral terminals in the topographically appropriate position at the dorsal margin of the dLGN was observed. C, Upon quantification, the ratio of ipsilateral-to-contralateral label showed a significant decrease in Ten-m2 KOs $(n=3)$, compared with WTs $(n=5)$. ret, retina; dm, dorsomedial; vl, ventrolateral; $d$, dorsal; t, temporal; $n$, nasal; v, ventral. Scale bar, $200 \mu m$. ${ }^{*} p<0.05$, Mann-Whitney U test.

the same animals. Further, while similar in overall shape, binocular responses appeared smaller in magnitude on average compared with those in WTs $(n=10)$, with a significant reduction being found in peak-to-trough magnitude (Fig. 10C, $D ; p=$ 0.040 , Student's unpaired $t$ test), with a trend toward a decrease also seen in the AUC ( $p=0.077$, Student's unpaired $t$ test). Further, a significantly smaller rise rate was found for the peak component in KOs ( $p=0.011$, Student's unpaired $t$ test). This corresponded with a significant reduction in peak magnitude ( $p=0.012$, Student's unpaired $t$ test), which occurred in the absence of a change in peak latency ( $p=0.688$, Student's unpaired $t$ test). A similar trend was found for the negative component, which exhibited a decreased slope $(p=0.078$, Student's unpaired $t$ test), despite a nonsignificant decrease in trough magnitude ( $p=0.203$, Student's unpaired $t$ test) and no difference in trough latency ( $p=0.398$, Student's unpaired $t$ test). All VEP data for binocular and monocular responses are summarized in Table 1 (statistical analyses were performed using two-tailed Student's unpaired $t$ tests with Welch correction to account for unequal variances).

Analysis of monocular responses revealed that the peak-totrough magnitude (Fig. $10 C, D ; p=0.298)$ and AUC $(p=0.675)$ for mean contralateral responses were slightly lower in Ten-m2 versus WT VEPs, although they were not significantly changed. Further analysis of peak $(p=0.444)$ and trough $(p=0.237)$ components also found no significant differences. Similarly, the slope of peak $(p=0.121)$ and peak-to-trough components $(p=$ 0.199 , Student's unpaired $t$ test), as well as latencies to peak ( $p=$ $0.110)$ and trough $(p=0.322)$, were not significantly different between genotypes for contralateral responses.

In contrast, ipsilateral responses exhibited a significant decrease in peak-to-trough magnitude (Fig. 10C,D; $p=0.033$ ) and AUC ( $p=0.023)$. While the magnitude of the peak component was significantly reduced in Ten-m2 KO ipsilateral responses $(p=0.034)$, this was not associated with a significant change in the peak slope $(p=0.714)$, or peak latency $(p=0.590)$. For the negative component, a nonsignificant trend toward reduction in trough magnitude $(p=0.070)$ was found, along with a similar reduction in peak-to-trough slope $(p=0.092)$. Like binocular responses, no change in trough latency was observed $(p=0.237)$, suggesting that the decreased slopes of binocular and ipsilateral $\mathrm{KO}$ responses were attributable to differences in the magnitude of each component.

Comparisons across genotypes further revealed a significant reduction in the ratio of ipsilateral-to-contralateral responses (comparing peak-to-trough magnitudes; Fig. 10E; mean \pm SEM; WT: $0.81 \pm 0.05$; Ten-m2 KO: $0.59 \pm 0.07 ; p=$ 0.022 , Student's unpaired $t$ test) in Ten-m 2 KO versus WT VEPs. While the contralateral-to-binocular response ratio (mean \pm SEM; WT: $0.79 \pm 0.05$; Ten-m2 KO: $0.94 \pm 0.07 ; p=$ 0.108 , Student's unpaired $t$ test) appeared higher in Ten-m2 KO VEPs, this was not significantly different to WTs. Similarly, no significant differences were observed between genotypes for the ipsilateral-to-binocular response ratio (mean \pm SEM; WT: $0.62 \pm$ 0.03; Ten-m2 KO: $0.55 \pm 0.07 ; p=0.393$, Student's unpaired $t$ test). These altered relationships become apparent when comparing average Ten-m2 KO binocular/monocular traces (Fig. 10F). Here, the average WT binocular response exhibited considerable alignment with averaged monocular traces: an indication of the contribution that each eye has to normal binocular activation. The alignment of KO traces, however, was less apparent for VEPs that were maximally responsive to stimuli presented at the pericentral portion of the binocular zone.

Contralateral VEPs recorded from medial V1 showed no significant differences for all parameters that were assessed above, with recordings from both genotypes ( $n=4$ for each genotype) being largely similar in overall shape, magnitude, and latency (data not shown). The similarity of the contralateral responses obtained from medial V1 indicates that deficits were specific to the binocular zone of Ten-m2 KO visual cortex. Together, these data support our c-fos experiments suggesting that ipsilateral in- 
A

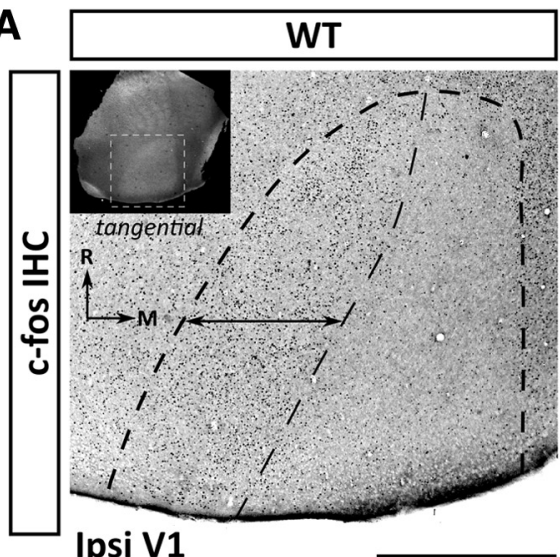

Ipsi V1

C



D
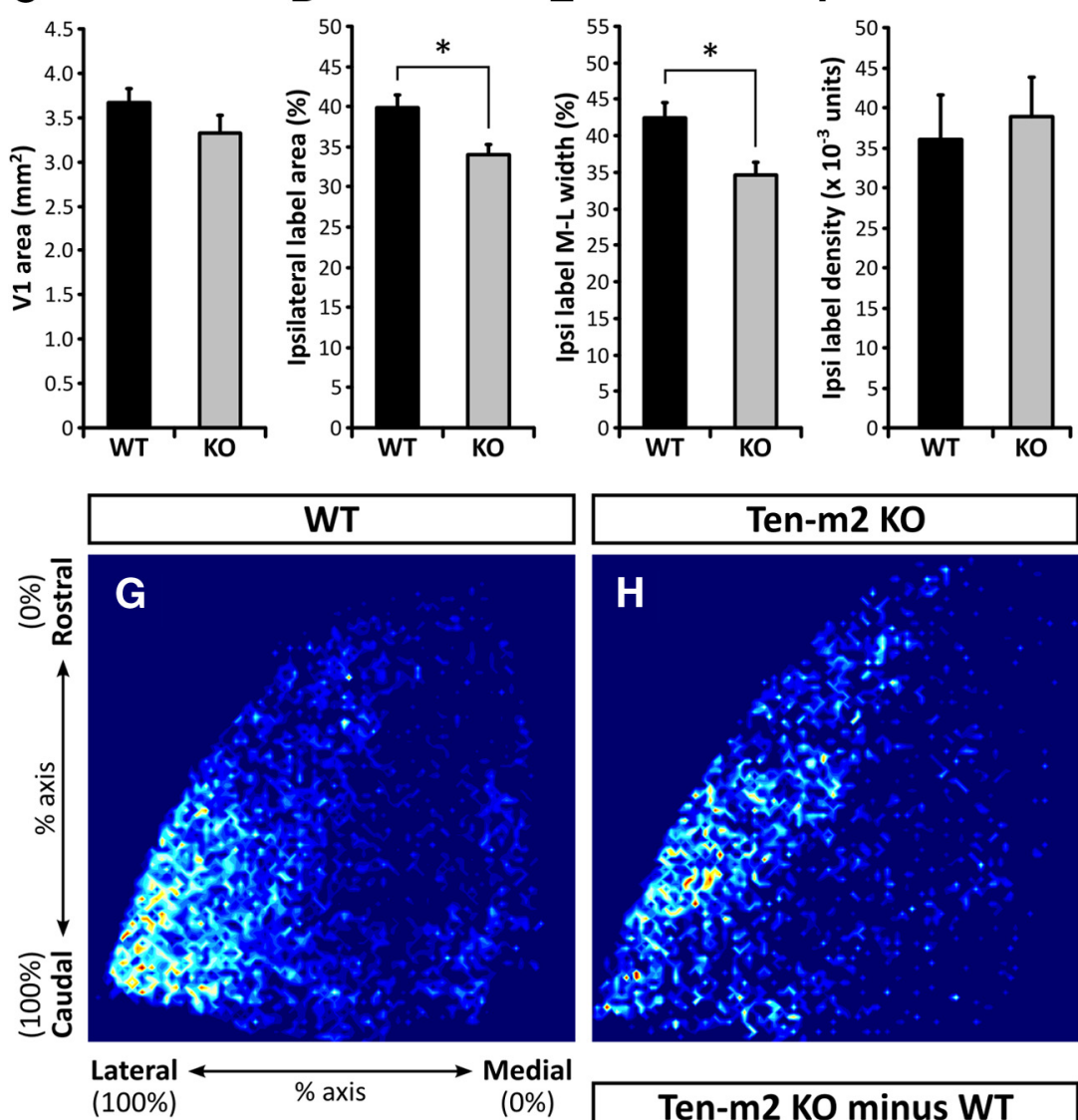

E
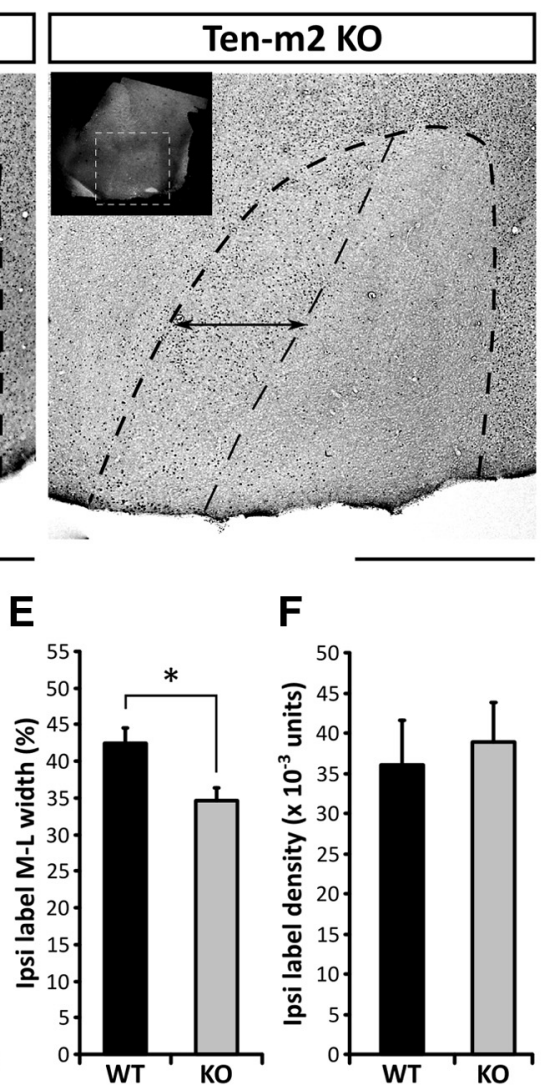

$\mathbf{F}$
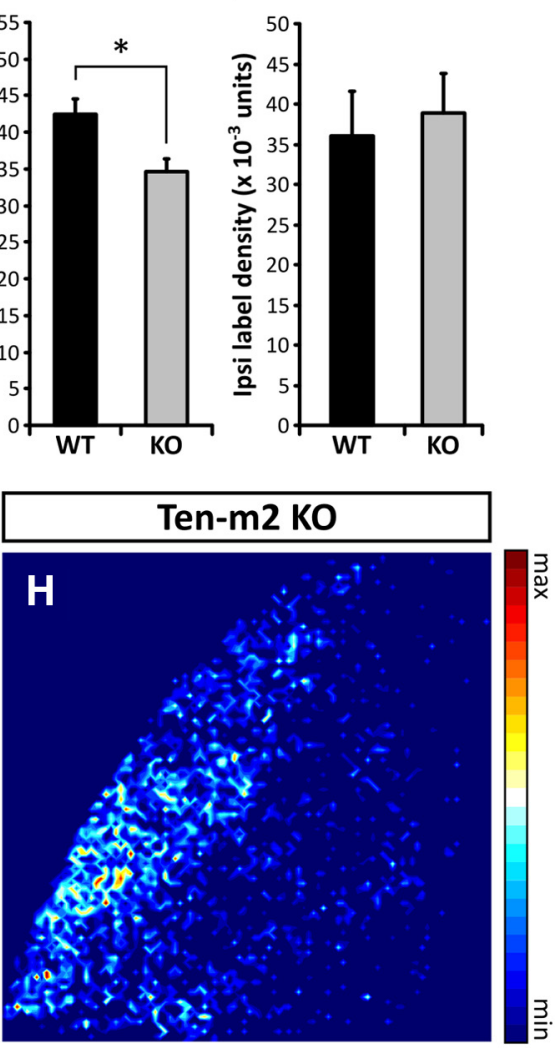

$0 \%$

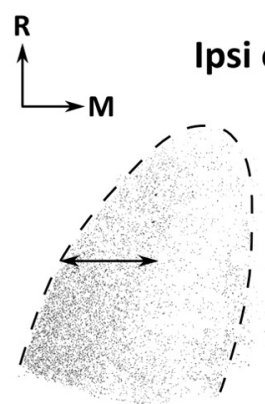

WT psi overlay

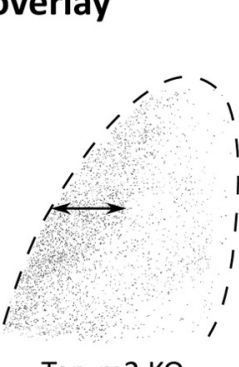

Ten-m2 KO

\section{Ten-m2 KO minus WT}

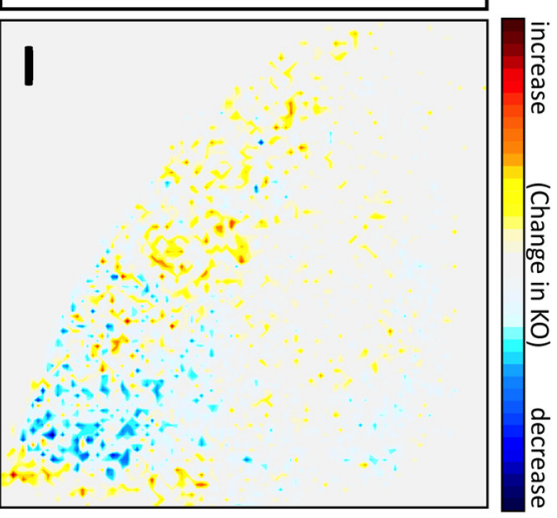

Figure 9. Evidence for a reduction in ipsilaterally driven cortical activity in V1 of Ten-m2 K0 mice. Ipsilateral to the active eye of monocularly inactivated WT $(\boldsymbol{A})$ and Ten-m2 K0 mice $(\boldsymbol{B})$, tangential sections through layer IV of V1 (dashed outline) showed high levels of c-fos immunostaining within the lateral-most region, along the entire rostrocaudal extent. Insets in $\boldsymbol{A}$ and $\boldsymbol{B}$ indicate the

puts to $\mathrm{V} 1$ are reduced in the absence of Ten-m2, contributing to an overall alteration to normal binocular activity.

Ten-m2 KO mice demonstrate an impaired ability to discriminate between dorsally located visual stimuli Our VEP results were consistent with reduced ipsilateral drive across the mediolateral axis of V1. This correlates well with the decrease of both the width of the retinal VTC and the binocular zone in V1. We were unable, however, to use VEPs to assess how the impact of the most pronounced anatomical phenotype in Ten-m2 KOs, namely the marked decrease in ipsilateral projections originating from ventral retina, affected visual function. To determine whether this change leads to a functional impairment in $\mathrm{KO}$ mice, we designed and developed a behavioral paradigm that specifically assessed their ability to discriminate between stimuli presented at dorsal locations in their visual field, based on the two alternate forced choice visual task developed by Prusky et al. (2000) (Fig. 11A).

Upon confirming that WT and $\mathrm{KO}$ mice were able to reach a predetermined performance threshold ( $\geq 80 \%$ correct over 10 consecutive trials) for the presentation of visual gratings at water level, we tested their ability to distinguish stimuli presented at increasingly higher locations within the dorsal visual field. The dorsal discrimination threshold (DDT) was determined to be the maximal height at

location of V1 identified from transillumination images (white dashed outline). Assessment of thresholded images revealed a reduction in the area occupied by c-fos-positive cells, as well as a slight narrowing along the mediolateral axis (double arrows) of this region in Ten-m2 KOs. While no significant difference in total tangential area of $\mathrm{V} 1$ was found between genotypes $(\boldsymbol{C})$, there was a significant decrease in both percentage area (D) and percentage width of ipsilateral label along the mediolateral V1 axis $(\boldsymbol{E})$ in Ten-m2 K0s $(n=6)$, compared with WTs $(n=4)$. Furthermore, the density of ipsilateral label $(\boldsymbol{F})$ was similar between genotypes. Images in $\boldsymbol{A}$ and $\boldsymbol{B}$ are representative tangential sections through V1 showing c-fos immunostaining. Rostral $(R)$ is to the top and medial $(M)$ to the right. Scale bars, $1 \mathrm{~mm} .{ }^{*} p<0.05$, Student's unpaired $t$ test. $\mathbf{G}, \boldsymbol{H}$, Composite heatmaps were generated from overlaid thresholded images (see bottom left). Regions in red indicate greater average levels of label across all animals, while dark blue regions indicate the absence of label. A higher proportion of label in WTs was observed within caudal V1, which in addition to the narrowing of mediolateral width, was notably absent in Ten-m2 K0 mice. I, Subtraction of WT signal from Ten-m2 K0 signal served to highlight these differences, with blue regions showing where label is decreased in $\mathrm{KO}$, with respect to WT. Signal is plotted along the percentage of the rostrocaudal and mediolateral axes of V1; color scale shown to the right. 
A
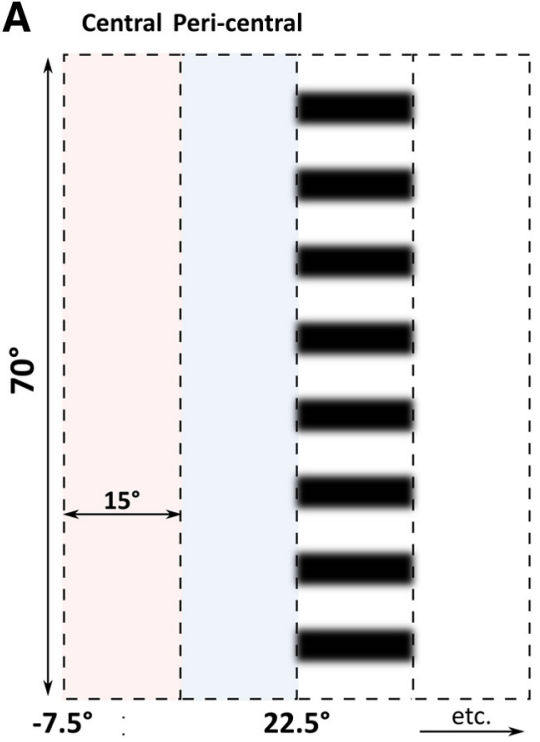

B
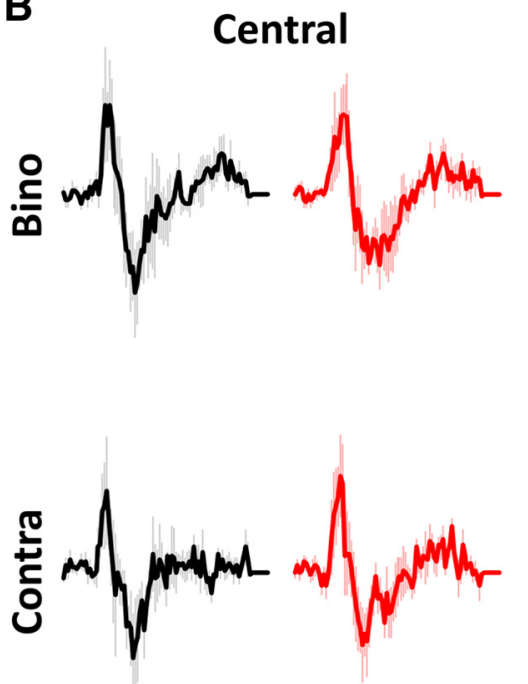

C

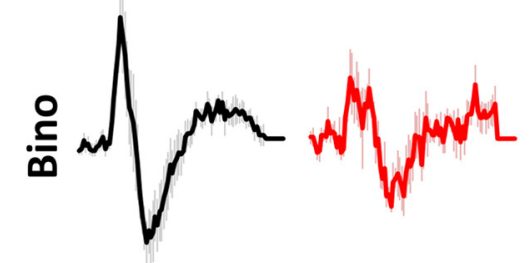

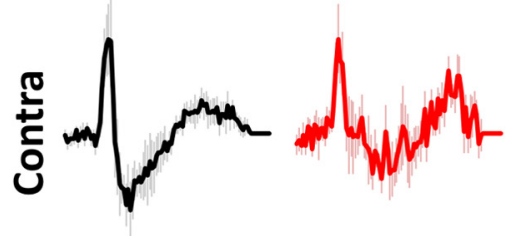

$0^{\circ}$
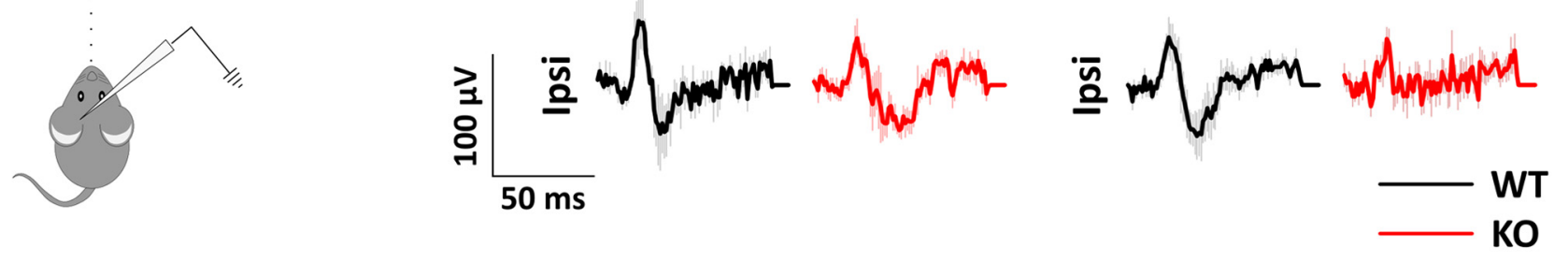

D

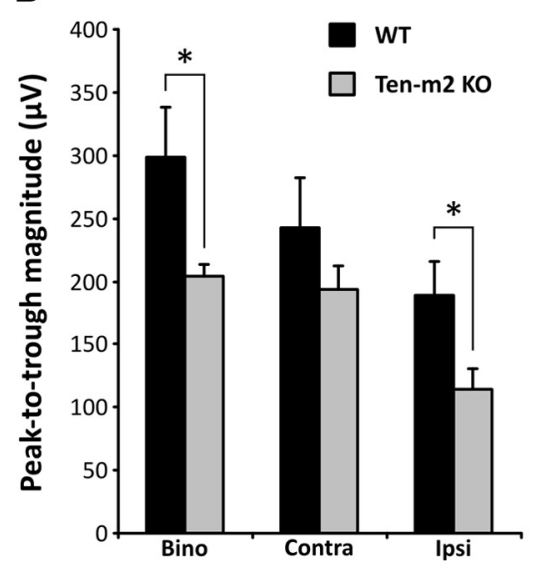

E



$\mathbf{F}$
Ten-m2 KO

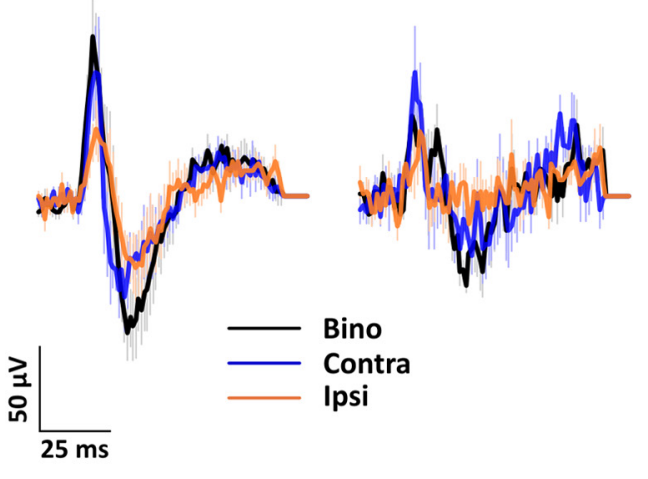

Figure 10. Differences in the level of activity observed across binocular (Bino), contralateral (Contra), and ipsilateral (Ipsi) VEP responses in Ten-m2 KO mice. $\boldsymbol{A}$, Individual VEP recordings were averaged and compared across binocular, contralateral, and ipsilateral presentations of visual stimuli (vertical columns of drifting horizontal sine-wave gratings). $\boldsymbol{B}$, For recordings made in lateral V1, stimuli situated centrally at the vertical meridian $\left(-7.5\right.$ to $7.5^{\circ}$; pink shaded region in $\left.A\right)$ resulted in no significant differences between WT $(n=5)$ and Ten-m2 KO $(n=5)$ recordings. $C$, Several differences were found, however, from those VEPs elicited from the adjacent peripheral location of the contralateral visual field (pericentral, $7.5-22.5^{\circ}$; blue shaded region in $\boldsymbol{A}$ ). $\boldsymbol{B}, \boldsymbol{C}$, Traces are averaged WT (black) and Ten-m2 KO (red) recordings with error bars indicating SEM. D, E, Quantification of pericentrally elicited VEPs revealed that while contralateral eye responses appeared unaltered, the magnitude (peak-to-trough) of binocular and ipsilateral VEPs (D) was significantly lower in Ten-m2 KOs $(n=6)$, compared with WTs $(n=10)$. This was further associated with a decrease in the ratio of ipsilateral-to-contralateral responses, when assessing peak-to-trough magnitude $(\boldsymbol{E})$. ${ }^{*} p<0.05$, Student's unpaired $t$ test. $\boldsymbol{F}$, A close alignment of averaged binocular (black), contralateral (blue), and ipsilateral (orange) responses was found for WT VEPs. This relationship was less evident for responses from Ten-m2 K0s.

which an animal was able to maintain a 70\% performance level (Fig. 11B). Based on this assessment, the DDT for WT mice was significantly higher than that of Ten-m2 KOs (mean DDT \pm SEM; WT: $17.7 \pm 1.0^{\circ}$, median: $18.4^{\circ}$, range: $11.3-21.8^{\circ}, n=11$; Ten-m2 KO: mean \pm SEM: $12.1 \pm 1.0^{\circ}$, median: $11.3^{\circ}$; range: 7.6-14. $9^{\circ}, n=9 ; \mathrm{U}=11.5, p=0.003$, Mann-Whitney $U$ test). Further analysis revealed that while individual variability existed, the number of animals that exhibited the highest quartile of DDT values was significantly greater for the WT group (animals scoring threshold heights in upper quartile, i.e., $\geq 25 \mathrm{~cm}$; WT: 7/11 animals, Ten-m2 KO: 0/9 animals; $p=0.004$, one-sided Fisher exact test). Conversely, the number of mice that maintained the lowest quartile of DDT levels was higher in Ten-m2 KOs compared with WTs (animals scoring in lower quartile, i.e., $\leq 15 \mathrm{~cm}$; WT: $1 / 11 ; 1 / 11$ animals; Ten-m2 KO: 5/9 animals; $p=0.038$, one-sided Fisher exact test). 
Table 1. Summary of VEP responses in WT and Ten-m2 KO mice

\begin{tabular}{|c|c|c|c|c|c|c|c|c|}
\hline Visual presentation & $\begin{array}{l}\mathrm{AUC}{ }^{a} \\
\left(\mu V^{*} \mathrm{~ms}\right)\end{array}$ & $\begin{array}{l}\text { Peak }^{b} \text {-to-trough } \\
\text { magnitude }(\mu \mathrm{V})\end{array}$ & $\begin{array}{l}\text { Peak slope } \\
(\mu \mathrm{V} / \mathrm{ms})\end{array}$ & $\begin{array}{l}\text { Peak magnitude } \\
(\mu \mathrm{V})\end{array}$ & $\begin{array}{l}\text { Time-to-peak } \\
\text { (ms) }\end{array}$ & $\begin{array}{l}\text { Peak-to-trough } \\
\text { slope ( } \mu \mathrm{V} / \mathrm{ms})\end{array}$ & $\begin{array}{l}\text { Trough magnitude } \\
(\mu \mathrm{V})\end{array}$ & $\begin{array}{l}\text { Time-to-trough } \\
\text { (ms) }\end{array}$ \\
\hline \multicolumn{9}{|l|}{ Binocular } \\
\hline WT & $2233 \pm 444$ & $298.8 \pm 38.9$ & $56.9 \pm 9.3$ & $173.0 \pm 20.5$ & $21.5 \pm 0.8$ & $-46.3 \pm 13.8$ & $-125.8 \pm 19.4$ & $33.2 \pm 1.9$ \\
\hline Ten-m2 K0 & $1258 \pm 249$ & $204.6 \pm 8.8^{*}$ & $26.4 \pm 4.2^{*}$ & $107.2 \pm 8.9^{*}$ & $22.3 \pm 1.6$ & $-18.0 \pm 4.6$ & $-97.3 \pm 8.4$ & $36.6 \pm 3.3$ \\
\hline \multicolumn{9}{|l|}{ Contralateral } \\
\hline WT & $1431 \pm 389$ & $242.2 \pm 40.3$ & $51.4 \pm 9.3$ & $133.1 \pm 20.5$ & $20.0 \pm 0.6$ & $-39.8 \pm 12.2$ & $-109.1 \pm 20.6$ & $32.7 \pm 3.6$ \\
\hline Ten-m2 K0 & $1217 \pm 313$ & $193.9 \pm 18.9$ & $30.7 \pm 8.4$ & $112.0 \pm 17.2$ & $22.5 \pm 1.2$ & $-19.8 \pm 8.4$ & $-81.8 \pm 7.0$ & $39.0 \pm 4.9$ \\
\hline \multicolumn{9}{|l|}{ Ipsilateral } \\
\hline WT & $1249 \pm 256$ & $189.3 \pm 26.9$ & $24.8 \pm 4.0$ & $92.8 \pm 12.2$ & $22.1 \pm 1.0$ & $-24.0 \pm 6.7$ & $-96.5 \pm 17.6$ & $34.2 \pm 2.1$ \\
\hline Ten-m2 K0 & $452 \pm 176^{*}$ & $114.5 \pm 16.6^{*}$ & $29.0 \pm 10.3$ & $57.0 \pm 9.2^{*}$ & $24.2 \pm 3.5$ & $-9.5 \pm 4.4$ & $-57.5 \pm 8.9$ & $40.7 \pm 4.6$ \\
\hline
\end{tabular}

VEP data presented as mean \pm SEM. Asterisk indicates significant differences ( $p<0.05$, Student's unpaired $t$ test) in Ten-m2 KO responses $(n=6)$ compared to WT ( $n=10)$. ${ }^{a}$ AUC was calculated over the whole response. ${ }^{b}$ Peak refers to the maximum of the major positive component of each response. 'Trough refers to the minimum of the negative component.

A
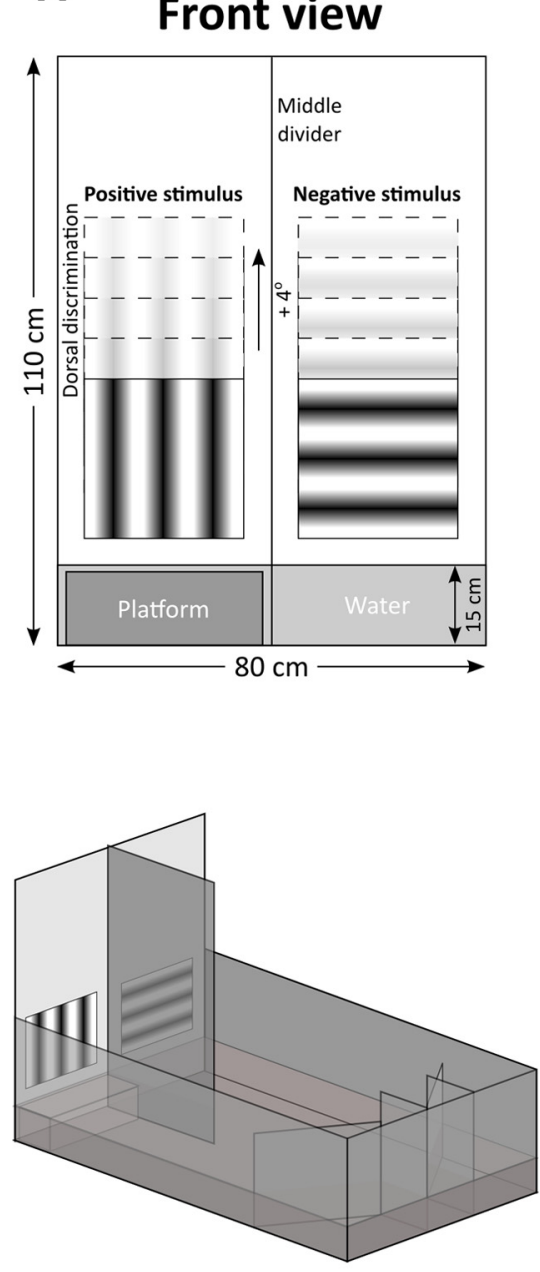

Top view

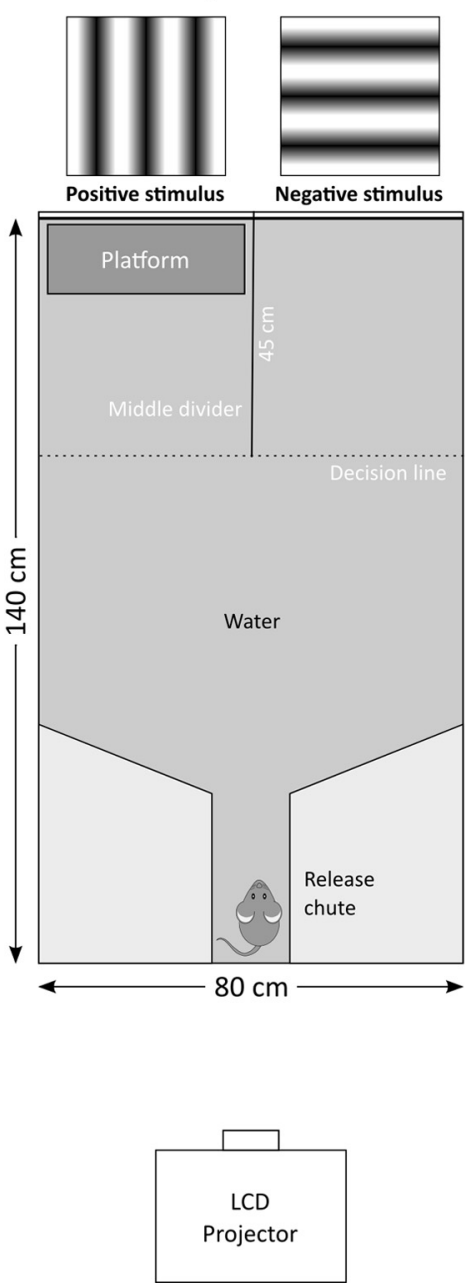

B
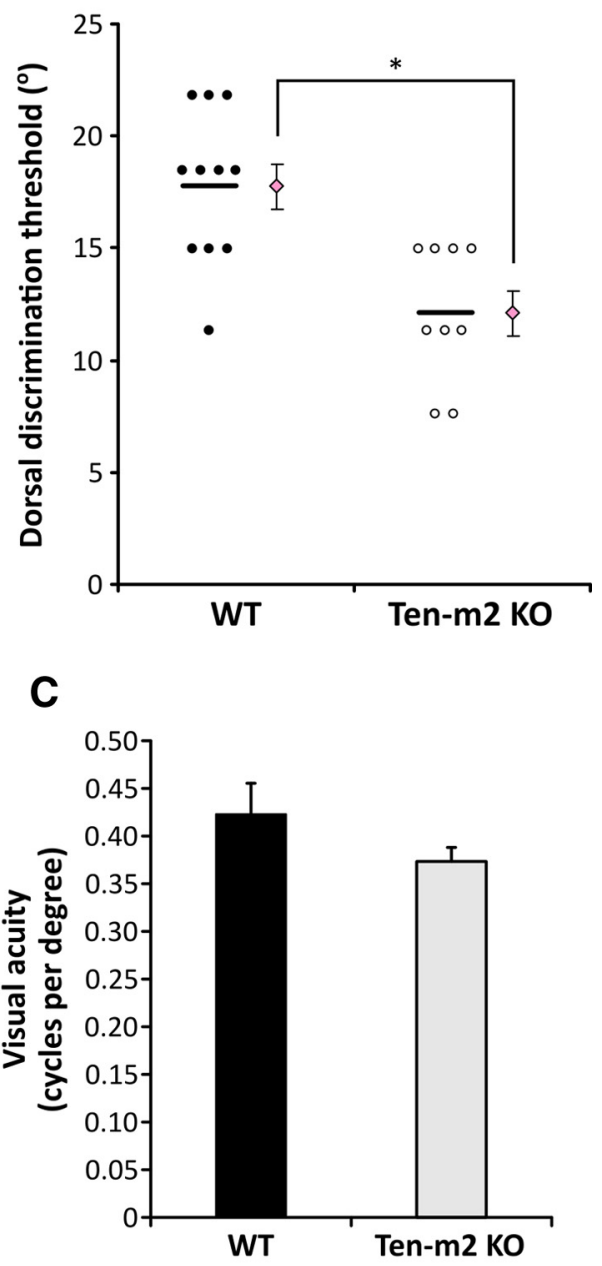

Figure 11. Behavioral evidence that the ability to discriminate in dorsal visual field is impaired in Ten-m2 K0 mice. $\boldsymbol{A}$, Schematic illustrating the swimming-based two-alternate visual discrimination task (adapted from Prusky et al., 2000) to challenge dorsal visual field of WT and Ten-m2 K0 mice. B, The mean and maximum dorsal discrimination threshold observed for Ten-m2 $\mathrm{K} 0$ mice was significantly lower than WTs, suggesting that Ten-m2 KOs were less able to discriminate between stimuli located at more dorsal positions. Furthermore, the minimum threshold was lower for Ten-m2 KOs than that found for WT mice. Threshold height at which the task could reliably be performed is plotted for individual WT (closed circles) and Ten-m2 KO (open circles) animals, alongside the mean for each genotype (bold line; diamond $\pm \mathrm{SEM} ; n=11 \mathrm{for}$ WT, $n=9$ for K0). ${ }^{*} p<0.005$, Mann-Whitney $U$ test. C, Visual acuity, assessed using this task, showed no significant difference between genotypes ( $n=4$ for each genotype; $p>0.05$, Mann-Whitney $U$ test).

Following the dorsal discrimination phase, some animals were further assessed for their visual acuity threshold (Fig. 11C). Here, stimuli of varying spatial frequencies were presented at water level. Threshold was again reached when the animal could not maintain a $70 \%$ performance level. We found that Ten-m2 KO mice had a slightly lower threshold for visual acuity compared with WT mice, although this difference was not significant (average visual acuity threshold \pm SEM; WT: $0.419 \pm 0.034$ cycles/ degree, median: 0.452 cycles/degree, range: $0.318-0.452$ cycles/ degree, $n=4$; Ten-m2 KO: $0.369 \pm 0.017$ cycles/degree, median: 0.352 cycles/degree, range: $0.352-0.419$ cycles/degree, $n=4 ; \mathrm{U}=$ $4.0, p=0.225$, Mann-Whitney $U$ test). No systematic difference 
in swimming trajectories was observed across genotypes, indicating that the KOs were not resolving the visual stimuli at different distances from the target compared with WTs (data not shown). Further, the data we obtained for WT mice fell within the range of visual acuity thresholds that have previously been reported for C57BL/6 mice using different variations of this task (0.375-0.510 cycles/degree; Prusky et al., 2000; Wong and Brown, 2006). This suggests that the modifications to the apparatus and test procedures that were used here did not significantly affect the ability of test animals to perform the task.

To confirm that the differences in performances observed between genotypes were not due to parameters other than the altered visual pathways exhibited by Ten-m2 KOs, a separate analysis was conducted to assess swimming behavior. Qualitative assessment yielded no observable differences between genotypes in overall motor coordination as well as head positioning while swimming. Moreover, measured head-angles during swimming in WTs and Ten-m2 KOs were largely comparable (mean \pm SEM; WT: $20.0 \pm 0.4^{\circ}, n=$ 4; Ten-m2 KO: $18.9 \pm 0.3^{\circ}, n=4 ; p=0.072$, Student's unpaired $t$ test). The potential for a very minor difference in head position (approx $1^{\circ}$ ) suggested by these data is not enough to accommodate the significant differences in effective DDTs observed between the two groups. Further, no obvious differences in eye position, interocular distances (mean \pm SEM; WT: $10.94 \pm 0.16 \mathrm{~mm}, n=4$; Ten-m2 KO: $10.73 \pm 0.14 \mathrm{~mm}, n=4 ; p=0.353$, Student's unpaired $t$ test; see also Fig. $2 C, D$ ) or other gross anatomical features that might impact the ability of the animals to perform the task were noted between genotypes. This suggests that the observed differences in performance associated with dorsal visual field were likely specific to visual deficits in Ten-m2 $\mathrm{KO}$ mice.

\section{Discussion}

We show that Ten- $\mathrm{m} 2$ regulates the size and location of ipsilateral retinal projections to the SC and dLGN: its deletion produces coordinated changes in laterality and mapping. These modifications are most marked in ventral retina, causing functional deficits including impaired performance on a visual discrimination task. Together with previous work, our results suggest complementary roles for multiple Ten-ms in the formation of binocular circuits.

\section{Ten-m molecules exhibit multiple and distinct roles in the development of the mouse visual system}

We demonstrate that Ten-m2 is expressed in the retina, dLGN, SC, and V1. Expression is uniform in these areas, complementing the graded expression of Ten-m3 (high in regions corresponding to ventral retina; Leamey et al., 2007; Dharmaratne et al., 2012). Multiple teneurins in interconnected visual areas have been reported in chicks, where nonoverlapping Ten-m 1 and Ten-m 2 expression distinguishes between tectofugal and thalamofugal pathways, while a mapping role is proposed for Ten-m4 (Kenzelmann et al., 2008; Kenzelmann-Broz et al., 2010). The distinction between mouse and chick in Ten-m/ teneurin expression patterns and roles may reflect species differences in binocularity and/or downstream molecular pathways linked to Ten-m/teneurins, as suggested for other conserved molecules (Carreres et al., 2011).

We found that Ten-m2 is critical for the development of the ipsilateral retinal pathway, in a manner distinct to Ten-m3 (Leamey et al., 2007). Our data suggest that Ten- $\mathrm{m} 2$ promotes a coordinated ipsilateral midline-guidance and mapping program for a subregion of the VTC. This contrasts with Ten-m3, which has a dramatic impact on mapping of the entire ipsilateral projection, with no obvious impact on laterality (Leamey et al., 2007).

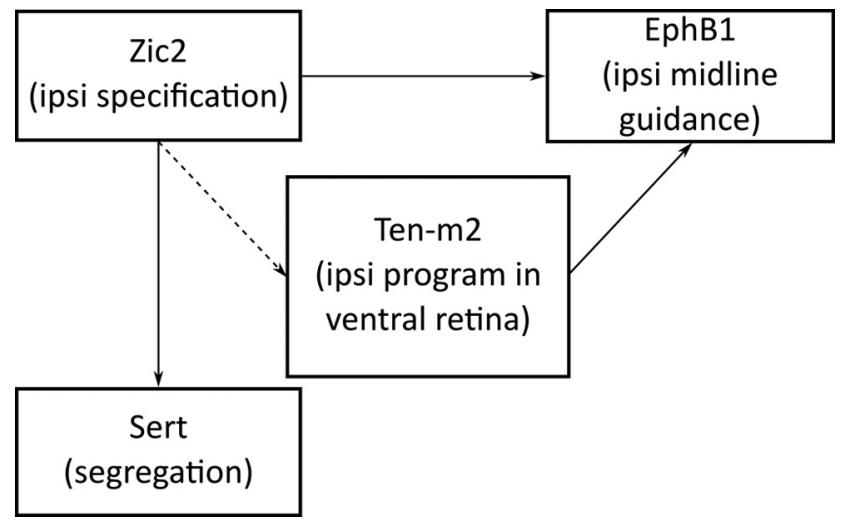

Figure 12. Schematic diagram illustrating gene interactions hypothesized to regulate the formation of the ipsilateral retinofugal pathway. Zic2 specifies ipsilateral fate by driving EphB1 expression (arrow). EphB1 expression causes axons to project ipsilaterally at the chiasmatic midline. In the ventral retina, Ten-m2 is also required for EphB1 expression (arrow) and converts a contralateral mapping program to an ipsilateral one in this region. This may occur downstream, or independently, of Zic2 expression (dotted arrow). Further, Zic2 drives Sert expression to regulate segregation (arrow). This interaction is independent of the potential Zic2-Ten-m2EphB1 pathway. Arrows may indicate direct or indirect regulation (schema based on data in Herrera et al., 2003, Williams et al., 2003; García-Frigola et al., 2008; Rebsam et al., 2009, 2012; García-Frigola and Herrera, 2010 as well as current study).

\section{Ten- $\mathrm{m} 2$ promotes a coordinated ipsilateral-specific midline-} guidance and mapping role

The phenotype of Ten-m 2 KOs, with a $40 \%$ decrease in the size of the ipsilateral retinal projection, somewhat resembles other mutants where an ipsilateral determinant has been altered. Albino mice, and those deficient for Zic2, EphB1, or the sonic hedgehog receptor, Boc, all display a reduced ipsilateral population (Dräger and Olsen, 1980; Herrera et al., 2003; Williams et al., 2003; Fabre et al., 2010). Our data are unique, however, in that we see regional changes, with the ventral part of the ipsilateral projection predominantly affected. Topographically corresponding changes in both the dLGN and SC suggest a common retinal mechanism. Furthermore, our focal tracing experiments demonstrate that Ten- $\mathrm{m} 2$ is not required for the mapping of the contralateral or remnant ipsilateral projections: instead it converts a contralateral guidance and mapping program to an ipsilateral-specific one.

The ratio of ipsilateral-to-contralateral projections arising from ventral retina is decreased in Ten-m2 KOs. Although the reduced ipsilateral projection was independent of modifications in retinal Zic2 levels, it correlated with decreased EphB1 expression. In EphB1 KOs, Zic2-positive cells are misrouted contralaterally at the chiasm but map in a manner appropriate for ipsilateral axons, although they remain segregated from the remnant ipsilateral projection (Rebsam et al., 2009). Albino mice have reduced Zic2 and EphB1 expression, and the expanded contralateral projection maps in a manner topographically similar to the main contralateral projection, but remains segregated from it (Rebsam et al., 2012). Changes in Sert expression, a target of Zic2, which acts independently of EphB1, may regulate this segregation (García-Frigola and Herrera, 2010). Our data imply that in Ten-m2 KOs, there is a ventral population of Zic2-expressing RGCs that lack EphB1. These give rise to an expanded projection to contralateral dLGN, which exhibits normal topography and segregation, indicating a coordinated switch of normally ipsilateral RGCs to a contralateral fate. We propose, therefore, that Ten-m 2 acts downstream of Zic2 expression, upstream of EphB1, and independently of Sert (yet to be directly assessed) to regulate RGC fate (Fig. 12), repressing a contralateral program and/or 
promoting ipsilateral gene expression without impacting segregation. Since evidence for interactions between the cleavable intracellular domain (ICD) of chick Ten-m2 and Zic1 have been reported in vitro (Bagutti et al., 2003), direct interactions with Zic2 to regulate transcription of specific downstream targets are feasible. Alternatively, Ten-m2 may act independently of Zic2. It should be noted that EphB1 is expressed in ipsilaterally projecting RGCs cells that are never Zic2 positive (Herrera et al., 2003; Williams et al., 2003), indicating that other components of the ipsilateral specification pathway remain to be determined. Additional studies are needed to clarify the relationship between Zic2, Ten-m2, and EphB1. It will also be important to determine whether other laterality-defined markers (Pak et al., 2004; Williams et al., 2006; García-Frigola and Herrera, 2010) are altered in Ten-m2 KOs.

\section{Absence of Ten-m2 leads to region-specific changes to the ipsilateral projection}

It is intriguing that uniform Ten-m2 expression in the retina does not align specifically with the VT location of ipsilateral projections, nor does it closely correspond to the specific deficits seen in Ten-m2 KOs. How could the knockdown of Ten-m2 throughout the visual system cause the loss of the ipsilateral projection from a subregion of retina?

Ten-m3 expression is also nonspecific for the ipsilateral projection, yet Ten-m3 KOs display a dramatic disruption of ipsilateral targeting, with only a subtle impact on contralateral axons (Leamey et al., 2007; Dharmaratne et al., 2012). This suggests that both Ten-m 2 and Ten-m 3 act via interactions with other molecules. While interactions with molecules that specify laterality are likely, an additional scenario is that multiple Ten-ms have complementary/combinatorial roles in shaping the ipsilateral projection. This could be facilitated via heterodimerization and/or heterophilic interactions (Feng et al., 2002; Hong et al., 2012; Mosca et al., 2012). For Ten-m2, overlap with regionally expressed molecules [e.g., ventral Vax2, temporal FoxD1, ventral Ten-m3, temporal Zic4 (Mui et al., 2002; Leamey et al., 2007; Horng et al., 2009; Carreres et al., 2011) could explain its graded requirement in specifying $\mathrm{RGC}$ fate. Whether this occurs through direct binding with extracellular partners and/or signaling via its ICD, as well as the identity of the participants of such interactions, is of particular interest.

\section{Visual deficits arising from neuroanatomical changes in $\mathrm{KO}$ mice highlight important roles of Ten- $\mathrm{m} 2$ in binocular vision} The performance of Ten-m 2 KOs on our visual discrimination task, as well changes in binocular and ipsilateral VEPs, shows that loss of ipsilateral projections leads to impaired binocular visual function.

The diminished binocular and ipsilateral VEPs observed from pericentral visual field are to some extent unexpected, as they correspond to the largely preserved ipsilateral projection from temporal retina. The decrease in the radial dimension of the ipsilateral RGC population, however, may underlie this. Notably, these changes correlate with the decreased width of the binocular zone in the c-fos data indicating reduced ipsilateral drive in Ten-m2 KOs.

It is not possible to separate the functional impact of the reduced ipsilateral and expanded contralateral projections. We observed no change in the size of contralateral VEPs, while ipsilateral and binocular responses were significantly reduced, suggesting that the main functional impact of Ten- $\mathrm{m} 2$ deletion is due to the loss of ipsilateral projections. The possibility that there may be suppressive interactions between the expanded contralateral projections, and the preserved ipsilateral and contralateral pro- jections, despite their apparently normal topography, must be considered. This could arise due to a mismatch of ipsilateral and contralateral inputs in binocular regions within V1, which map specifically to the pericentral visual field, as for Ten-m3 KOs (Leamey et al., 2007; Merlin et al., 2013). How the combined effect of diminished ipsilateral, and expanded contralateral, input leads to this potential suppression has yet to be determined.

Interestingly, c-fos immunohistochemistry provided further evidence of a deficit in caudal V1, corresponding topographically with changes in ventral retina. We were unable, however, to confirm this with VEPs due to the difficulty in acquiring reliable responses from this region (Dräger and Olsen, 1980). Therefore, this was assessed behaviorally.

We are unaware of previous studies in which the dorsal visual field of mice has been systematically examined using a behavioral test performed on awake, freely moving animals. Various measures were taken to assure that our assessment was specific to dorsal visual field. Extraneous cues such as water surface reflections were eliminated. Potential nonvisual reasons for performance differences including swimming ability/trajectories, head angle, interocular distance, and the ability to acquire the task were compared and found not to differ between genotypes. The impaired performance on this task, therefore, was most likely related to the decrease in ipsilateral projections in Ten-m2 KOs, consistent with our VEP and c-fos data.

Whether the observed deficits are associated with changes to synaptic function and organization (Silva et al., 2011; Mosca et al., 2012) remains to be determined. Although Ten-m2 is expressed throughout the visual system, the largely normal contralateral responses argue that the impact of Ten-m 2 deletion is highly localized, and on its own has a relatively limited impact on synaptic function. It is possible that other Ten-ms may compensate: both Ten-m3 and Ten-m4 are expressed throughout the visual system (Leamey et al., 2007; 2008), although Ten-m1 is not (T.R. Young and C.A. Leamey, unpublished observations).

In conclusion, we propose that Ten- $\mathrm{m} 2$ acts to coordinate both laterality and mapping in the ventral retina via interactions with other regionally expressed molecules. Its deletion causes loss of ipsilateral drive and leads to defects in binocular vision.

\section{References}

Bagutti C, Forro G, Ferralli J, Rubin B, Chiquet-Ehrismann R (2003) The intracellular domain of teneurin-2 has a nuclear function and represses zic-1-mediated transcription. J Cell Sci 116:2957-2966. CrossRef Medline

Bovolenta P, Mason C (1987) Growth cone morphology varies with position in the developing mouse visual pathway from retina to first targets. J Neurosci 7:1447-1460. Medline

Brown LY, Kottmann AH, Brown S (2003) Immunolocalization of Zic2 expression in the developing mouse forebrain. Gene Expr Patterns 3:361-367. CrossRef Medline

Carreres MI, Escalante A, Murillo B, Chauvin G, Gaspar P, Vegar C, Herrera E (2011) Transcription factor foxd 1 is required for the specification of the temporal retina in mammals. J Neurosci 31:5673-5681. CrossRef Medline

Chan SO, Guillery RW (1994) Changes in fiber order in the optic nerve and tract of rat embryos. J Comp Neurol 344:20-32. CrossRef Medline

Dharmaratne N, Glendining KA, Young TR, Tran H, Sawatari A, Leamey CA (2012) Ten-m3 is required for the development of topography in the ipsilateral retinocollicular pathway. PLoS One 7:e43083. CrossRef Medline

Dräger UC (1975) Receptive fields of single cells and topography in mouse visual cortex. J Comp Neurol 160:269-290. CrossRef Medline

Dräger UC (1985) Birth dates of retinal ganglion cells giving rise to the crossed and uncrossed optic projections in the mouse. Proc R Soc Lond B Biol Sci 224:57-77. CrossRef Medline

Dräger UC, Olsen JF (1980) Origins of crossed and uncrossed retinal pro- 
jections in pigmented and albino mice. J Comp Neurol 191:383-412. CrossRef Medline

Dragunow M, Robertson HA (1988) Localization and induction of c-fos protein-like immunoreactive material in the nuclei of adult mammalian neurons. Brain Res 440:252-260. CrossRef Medline

Fabre PJ, Shimogori T, Charron F (2010) Segregation of ipsilateral retinal ganglion cell axons at the optic chiasm requires the Shh receptor Boc. J Neurosci 30:266-275. CrossRef Medline

Feng K, Zhou XH, Oohashi T, Mörgelin M, Lustig A, Hirakawa S, Ninomiya Y, Engel J, Rauch U, Fässler R (2002) All four members of the Ten-m/ Odz family of transmembrane proteins form dimers. J Biol Chem 277: 26128-26135. CrossRef Medline

García-Frigola C, Herrera E (2010) Zic2 regulates the expression of Sert to modulate eye-specific refinement at the visual targets. EMBO J 29:3170-3183. CrossRef Medline

García-Frigola C, Carreres MI, Vegar C, Mason C, Herrera E (2008) Zic2 promotes axonal divergence at the optic chiasm midline by EphB1dependent and -independent mechanisms. Development 135:1833-1841. CrossRef Medline

Godement P, Salaün J, Imbert M (1984) Prenatal and postnatal development of retinogeniculate and retinocollicular projections in the mouse. J Comp Neurol 230:552-575. CrossRef Medline

Guillery RW, Mason CA, Taylor JS (1995) Developmental determinants at the mammalian optic chiasm. J Neurosci 15:4727-4737. Medline

Herrera E, Brown L, Aruga J, Rachel RA, Dolen G, Mikoshiba K, Brown S, Mason CA (2003) Zic2 patterns binocular vision by specifying the uncrossed retinal projection. Cell 114:545-557. CrossRef Medline

Hofbauer A, Dräger UC (1985) Depth segregation of retinal ganglion cells projecting to mouse superior colliculus. J Comp Neurol 234:465-474. CrossRef Medline

Hong W, Mosca TJ, Luo L (2012) Teneurins instruct synaptic partner matching in an olfactory map. Nature 484:201-207. CrossRef Medline

Horng S, Kreiman G, Ellsworth C, Page D, Blank M, Millen K, Sur M (2009) Differential gene expression in the developing lateral geniculate nucleus and medial geniculate nucleus reveals novel roles for Zic4 and Foxp2 in visual and auditory pathway development. J Neurosci 29:13672-13683. CrossRef Medline

Kenzelmann D, Chiquet-Ehrismann R, Leachman NT, Tucker RP (2008) Teneurin- 1 is expressed in interconnected regions of the developing brain and is processed in vivo. BMC Dev Biol 8:30. CrossRef Medline

Kenzelmann-Broz D, Tucker RP, Leachman NT, Chiquet-Ehrismann R (2010) The expression of teneurin-4 in the avian embryo: potential roles in patterning of the limb and nervous system. Int J Dev Biol 54;1509-16. Medline

LaVail JH, Nixon RA, Sidman RL (1978) Genetic control of retinal ganglion cell projections. J Comp Neurol 182:399-421. CrossRef Medline

Leamey CA, Glendining KA, Kreiman G, Kang ND, Wang KH, Fassler R, Sawatari A, Tonegawa S, Sur M (2008) Differential gene expression between sensory neocortical areas: potential roles for Ten_m3 and Bcl6 in patterning visual and somatosensory pathways. Cereb Cortex 18:53-66. CrossRef Medline

Leamey CA, Merlin S, Lattouf P, Sawatari A, Zhou X, Demel N, Glendining KA, Oohashi T, Sur M, Fässler R (2007) Ten_m3 regulates eye-specific patterning in the mammalian visual pathway and is required for binocular vision. PLoS Biol 5:e241. CrossRef Medline

Leamey CA, Van Wart A, Sur M (2009) Intrinsic patterning and experiencedependent mechanisms that generate eye-specific projections and binocular circuits in the visual pathway. Curr Opin Neurobiol 19:181-187. CrossRef Medline

Lee R, Petros TJ, Mason CA (2008) Zic2 regulates retinal ganglion cell axon avoidance of ephrinB2 through inducing expression of the guidance receptor EphB1. J Neurosci 28:5910-5919. CrossRef Medline

Li H, Bishop KM, O'Leary DD (2006) Potential target genes of EMX2 include Odz/Ten-M and other gene families with implications for cortical patterning. Mol Cell Neurosci 33:136-149. CrossRef Medline

Merlin S, Horng S, Marotte LR, Sur M, Sawatari A, Leamey CA (2013) Deletion of Ten-m3 induces the formation of eye dominance domains in mouse visual cortex. Cereb Cortex 23:763-774. CrossRef Medline

Mesulam MM (1978) Tetramethyl benzidine for horseradish peroxidase neurohistochemistry: a noncarcinogenic blue reaction product with superior sensitivity for visualizing neural afferents and efferents. J Histochem Cytochem 26:106-117. CrossRef Medline
Mosca TJ, Hong W, Dani VS, Favaloro V, Luo L (2012) Trans-synaptic Teneurin signalling in neuromuscular synapse organization and target choice. Nature 484:237-241. CrossRef Medline

Mui SH, Hindges R, O'Leary DD, Lemke G, Bertuzzi S (2002) The homeodomain protein Vax2 patterns the dorsoventral and nasotemporal axes of the eye. Development 129:797-804. Medline

Muir-Robinson G, Hwang BJ, Feller MB (2002) Retinogeniculate axons undergo eye-specific segregation in the absence of eye-specific layers. J Neurosci 22:5259-5264. Medline

Oohashi T, Zhou XH, Feng K, Richter B, Mörgelin M, Perez MT, Su WD, Chiquet-Ehrismann R, Rauch U, Fässler R (1999) Mouse ten-m/Odz is a new family of dimeric type II transmembrane proteins expressed in many tissues. J Cell Biol 145:563-577. CrossRef Medline

Pak W, Hindges R, Lim YS, Pfaff SL, O'Leary DD (2004) Magnitude of binocular vision controlled by islet- 2 repression of a genetic program that specifies laterality of retinal axon pathfinding. Cell 119:567-578. CrossRef Medline

Péquignot MO, Provost AC, Sall é S, Taupin P, Sainton KM, Marchant D, Martinou JC, Ameisen JC, Jais JP, Abitbol M (2003) Major role of BAX in apoptosis during retinal development and in establishment of a functional postnatal retina. Dev Dyn 228:231-238. CrossRef Medline

Petros TJ, Shrestha BR, Mason C (2009) Specificity and sufficiency of EphB1 in driving the ipsilateral retinal projection. J Neurosci 29:3463-3474. CrossRef Medline

Porciatti V, Pizzorusso T, Maffei L (1999) The visual physiology of the wild type mouse determined with pattern VEPs. Vis Res 39:3071-3081. CrossRef Medline

Prusky GT, West PW, Douglas RM (2000) Behavioral assessment of visual acuity in mice and rats. Vis Res 40:2201-2209. CrossRef Medline

Rebsam A, Petros TJ, Mason CA (2009) Switching retinogeniculate axon laterality leads to normal targeting but abnormal eye-specific segregation that is activity dependent. J Neurosci 29:14855-14863. CrossRef Medline

Rebsam A, Bhansali P, Mason CA (2012) Eye-specific projections of retinogeniculate axons are altered in albino mice. J Neurosci 32:4821-4826. CrossRef Medline

Reese BE (1986) The topography of expanded uncrossed retinal projections following neonatal enucleation of one eye: differing effects in dorsal lateral geniculate nucleus and superior colliculus. J Comp Neurol 250:8-32. CrossRef Medline

Reese BE (1987) The position of the crossed and uncrossed optic axons, and the nonoptic axons, in the optic tract of the rat. Neuroscience 22:1025-1039. CrossRef Medline

Reese BE, Jeffery G (1983) Crossed and uncrossed visual topography in dorsal lateral geniculate nucleus of the pigmented rat. J Neurophysiol 49:877885. Medline

Reese BE, Guillery RW, Mallarino C (1992) Time of ganglion cell genesis in relation to the chiasmatic pathway choice of retinofugal axons. J Comp Neurol 324:336-342. CrossRef Medline

Silva JP, Lelianova VG, Ermolyuk YS, Vysokov N, Hitchen PG, Berninghausen O, Rahman MA, Zangrandi A, Fidalgo S, Tonevitsky AG, Dell A, Volynski KE, Ushkaryov YA (2011) Latrophilin 1 and its endogenous ligand Lasso/teneurin-2 form a high-affinity transsynaptic receptor pair with signaling capabilities. Proc Natl Acad Sci U S A 108:12113-12118. CrossRef Medline

Tian NM, Pratt T, Price DJ (2008) Foxg1 regulates retinal axon pathfinding by repressing an ipsilateral program in nasal retina and by causing optic chiasm cells to exert a net axonal growth-promoting activity. Development 135:4081-4089. CrossRef Medline

Trevelyan AJ, Upton AL, Cordery PM, Thompson ID (2007) An experimentally induced duplication of retinotopic mapping within the hamster primary visual cortex. Eur J Neurosci 26:3277-3290. CrossRef Medline

Tucker RP, Beckmann J, Leachman NT, Schöler J, Chiquet-Ehrismann R (2012) Phylogenetic analysis of the teneurins: conserved features and premetazoan ancestry. Mol Biol Evol 29:1019-1029. CrossRef Medline

Wagor E, Mangini NJ, Pearlman AL (1980) Retinotopic organization of striate and extrastriate visual cortex in the mouse. J Comp Neurol 193: 187-202. CrossRef Medline

Wang Q, Burkhalter A (2007) Area map of mouse visual cortex. J Comp Neurol 502:339-357. CrossRef Medline

Williams SE, Mann F, Erskine L, Sakurai T, Wei S, Rossi DJ, Gale NW, Holt CE, Mason CA, Henkemeyer M (2003) Ephrin-B2 and EphB1 mediate 
retinal axon divergence at the optic chiasm. Neuron 39:919-935. CrossRef Medline

Williams SE, Grumet M, Colman DR, Henkemeyer M, Mason CA, Sakurai T (2006) A role for Nr-CAM in the patterning of binocular visual pathways. Neuron 50:535-547. CrossRef Medline

Wong AA, Brown RE (2006) Visual detection, pattern discrimination and visual acuity in 14 strains of mice. Genes Brain Behav 5:389-403. CrossRef Medline
Young TR, Leamey CA (2009) Teneurins: important regulators of neural circuitry. Int J Biochem Cell Biol 41:990-993. CrossRef Medline

Zhou XH, Brandau O, Feng K, Oohashi T, Ninomiya Y, Rauch U, Fässler R (2003) The murine Ten-m/Odz genes show distinct but overlapping expression patterns during development and in adult brain. Gene Expr Patterns 3:397-405. CrossRef Medline 